\title{
Geometric Quantization of the BRST Charge
}

\author{
G.M. Tuynman ${ }^{\star}$ \\ UFR de Mathématiques, Université de Lille I, 59655 Villeneuve d'Ascq Cedex, France
}

Received July 12, 1989; in revised form February 21, 1992

\begin{abstract}
In the first half of this paper (Sects. 1-4) we generalise the standard geometric quantization procedure to symplectic supermanifolds. In the second half (Sects. 5, 6) we apply this to two examples that exhibit classical BRST symmetry, i.e., we quantize the BRST charge and the ghost number. More precisely, in the first example we consider the reduced symplectic manifold obtained by symplectic reduction from a free group action with $\mathrm{Ad}^{*}$-equivariant moment map; in the second example we consider a foliated configuration space, whose cotangent bundle admits the construction of a BRST charge associated to this foliation. We show that the classical BRST symmetry can be described in terms of a hamiltonian supergroup action on the extended phase space, and that geometric quantization gives us a super-unitary representation of this supergroup. Finally we point out how these results are related to reduction at the quantum level, as compared with the reduction at the classical level.
\end{abstract}

\section{Introduction and Summary}

Recently there has been much interest at the classical level in the relation between reduced/constrained systems and the original (or extended) systems, especially with regard to the Poisson algebras. One considers a classical physical system described by a phase space $\left(M_{0}, \omega_{0}\right)$ (a symplectic manifold) subject to constraint functions $J_{a}=0$, which we will assume to be first class. In an abstract way the actual phase space (also called the reduced phase space) is easy to describe. One considers the constraint set $C=\left\{m \in M_{0} \mid \forall a J_{a}(m)=0\right\}$ and the restriction of $\omega_{0}$ to $C$. The leaves of the characteristic foliation $\mathscr{D}_{\text {char }}$ of $\left.\omega_{0}\right|_{C}$ represent gauge equivalent points of this physical system and the quotient $M_{r}=C / \mathscr{D}_{\text {char }}$ gives us the actual phase space of the system. In favorable circumstances $M_{r}$ is a (symplectic) manifold. If the constraint functions are derived from a proper symplectic group action on $\left(M_{0}, \omega_{0}\right)$, this is the well known Marsden Weinstein reduction [MW].

\footnotetext{
* Research supported by the Dutch Organization for Scientific Research (NWO); E-mail: gmt@gat.citilille.fr
} 
One of the goals of the classical BRST symmetry approach (see e.g., [BM, He, HT, FHST, Sta1, Sta2, KS, Lo, DET] and references therein) is to give a similar description of the reduced Poisson algebra $C^{\infty}\left(M_{r}\right)$, i.e. to describe it as a quotient $A / I$, where $A$ is a subalgebra of a Poisson algebra defined by equations, and where $I$ is an ideal in $A$. Apart from an intrinsic interest, the "why" of this elaborate attention is the idea that it might be difficult to quantize the reduced system itself. Hence one would like to quantize before reduction, or in other words to apply the constraints after the quantization. It follows that one would like to have some kind of evidence/proof that the results so obtained are in agreement with the direct quantization of the reduced system (if feasible).

The first idea on how to apply the constraints after quantization, already given by Dirac, is that if the phase space $\left(M_{0}, \omega_{0}\right)$ is quantized by the Hilbert space $\mathscr{H}_{0}$ and the constraint functions $J_{a}$ by self-adjoint operators $\tau\left(J_{a}\right)$ on $\mathscr{H}_{0}$, then the quantization of the reduced system is given by the (Hilbert) space $\mathscr{H}_{\text {red }}=$ $\left\{\psi \in \mathscr{H}_{0} \mid \forall a \tau\left(J_{a}\right) \psi=0\right\}$. One immediate problem with this approach is that it not clear how to quantize classical observables of the reduced system. Moreover, it has been shown (e.g. [DEGT, DEGST]) that in general this approach to the quantization of the reduced system $M_{r}$ does not agree with any reasonable direct quantization of $M_{r}$.

The above described procedure of reduction after quantization is based on the idea that one should compare the classical phase space with the quantum Hilbert space. If we shift our point of view to the idea that the quantum Hilbert space is in some sense a representation space for the Poisson algebra, then we get a whole new approach to reduction after quantization (note that this viewpoint is carried out in extrema in the deformation/star-product approach to quantization where one foregoes the Hilbert space altogether). Now the BRST symmetry is essentially a symmetry at the level of the Poisson algebras rather than of the underlying symplectic manifolds (although we will show that in our examples it is induced by a group action on a symplectic manifold). It follows that with our new point of view the classical BRST symmetry will play an important role in the reduction after quantization. It is the aim of this paper to investigate how the classical BRST symmetry is related to a consistent reduction after quantization, where consistent means that we want to apply quantum constraints in such a way that the results are equivalent to a direct quantization of the reduced classical system.

Since the classical BRST symmetry involves odd-degrees of freedom (or in other words supermanifolds), we have to have a well defined procedure to quantize these. To that extent we take the geometric quantization procedure and we adapt it (Sects. 2-4) to symplectic supermanifolds in order to have a consistent quantization method which applies to all kinds of degrees of freedom in the same way at the same time. In Sect. 2 we adapt the notions of frame bundles and densities to supermanifolds; in Sect. 3 we use these notions to adapt the geometric quantization procedure to supermanifolds, and in Sect. 4 we apply the adapted geometric quantization procedure to a very simple example containing only odd degrees of freedom. The, for this summary basic, idea of geometric quantization is that a phase space $M$ together with a polarization $\mathscr{P}$ determine (in nice cases) a representation space $\Gamma(\mathrm{QB})^{\mathscr{P}}$ for (a part of) the Poisson algebra $C^{\infty}(M)$ with $\tau$ the map from $C^{\infty}(M)$ to operators on $\Gamma(\mathrm{QB})^{\mathscr{P}}$. Usually there exists a natural subspace $\Gamma_{c}(\mathrm{QB})^{\mathscr{P}}$ of $\Gamma(\mathrm{QB})^{\mathscr{P}}$ which carries a scalar product such that the operators $\tau(f)$ are self-adjoint; the Hilbert space of quantum mechanics then is supposed to be the completion of $\Gamma_{c}(\mathrm{QB})^{\mathscr{P}}$. 
Although Sects. 2-4 contain essential basic material, the knowledgeable reader may skip it over initially, referring back as necessary. In Sects. 5 and 6 we then discuss two applications of the adapted geometric quantization procedure, which both fit the same basic scheme. At the classical level we assume some regularity conditions, among which are the conditions that the constraint set $C \subset M_{0}$ has a well-defined codimension $n$, and that the reduced phase space $M_{r}$ is a smooth symplectic manifold. We then extend the phase space $M_{0}$ to a super phase space $M$ by adding $2 n$ odd directions (ghosts and anti-ghosts). On this extended phase space $M$ we construct a symplectic action $\Phi$ of a supergroup $S \subset S G L(1,1)$ whose conserved quantities are called the BRST-charge $\Omega$ and the total degree Td. Basically the action of $S$ can be described by saying that the even parameter in $S$ rescales the odd-directions (ghosts and anti-ghosts) and the odd-parameter moves a point of $M_{0}$ in a nilpotent direction which is given by the odd coordinates. Thus the action of $S$ on $M$ induces the trivial action on $M_{0}$. Since the functions $\Omega$ and Td satisfy the relations $\{\Omega, \Omega\}=0$ and $\{\mathrm{Td}, \Omega\}=\Omega$, one can define the so-called BRST cohomology,

$$
\begin{array}{r}
H_{\text {BRST }}^{k}=\left\{f \in C^{\infty}(M) \mid\{\mathrm{Td}, f\}=k \cdot f \&\{\Omega, f\}=0\right\} \text { modulo } \\
\left\{f=\{\Omega, g\} \mid g \in C^{\infty}(M) \&\{\mathrm{Td}, g\}=(k-1) \cdot g\right\} .
\end{array}
$$

The BRST cohomology in dimension zero, which can be described as equivalence classes of functions globally invariant under the $S$ action, inherits from $C^{\infty}(M)$ the structure of a Poisson algebra and there exists a natural morphism of Poisson algebras

$$
H_{\text {BRST }}^{0} \rightarrow C^{\infty}\left(M_{r}\right)
$$

We then apply the adapted geometric quantization procedure to the extended phase space $M$ and the functions Td and $\Omega$. It turns out that the operators $\tau(\mathrm{Td})$ and $\tau(\Omega)$ can be integrated to an action $U$ of $S$ on $\Gamma(\mathrm{QB})^{\mathscr{P}}$ which is super-unitary when restricted to $\Gamma_{c}(\mathrm{QB})^{\mathscr{P}}$. In "analogy" with the classical case we define a subspace $\Gamma_{\text {red }} \subset \Gamma(\mathrm{QB})^{\mathscr{P}}$ by the action of $S$ as

$$
\Gamma_{\text {red }}=\left\{\psi \in \Gamma(\mathrm{QB})^{\mathscr{P}} \mid \forall s \in S: U(s) \psi=\operatorname{Ber}(s)^{n / 2} \cdot \psi\right\},
$$

where Ber of a matrix is the Berezinian, also called the superdeterminant. We then show that functions belonging to the same equivalence class in $H_{\text {BRST }}^{0}$ induce the same operators on $\Gamma_{\text {red }}$ and hence that $\Gamma_{\text {red }}$ is a representation space for (a part of) the Poisson algebra $H^{0}{ }_{\text {BRST }}$. Finally we show (or quote the literature) that there exists a polarization $\mathscr{P}_{r}$ on $M_{r}$ with a quantization map $\tau_{r}$ from $C^{\infty}\left(M_{r}\right)$ to operators on $\Gamma\left(\mathrm{QB}_{r}\right)^{\mathscr{P}_{r}}$ with two nice properties. In the first place $\Gamma\left(\mathrm{QB}_{r}\right)^{\mathscr{P}_{r}}$ is (naturally) isomorphic to $\Gamma_{\text {red }}$. In the second place, using this isomorphism, we have the following commutative diagram

$$
\begin{gathered}
H_{\text {BRST }}^{0} \ni[f] \underset{\text { quantization }}{\longrightarrow} \tau(f) \text { operator on } \Gamma_{\text {red }} \\
\text { map (1.2) } \downarrow \\
\quad \downarrow \text { isomorphism } \Gamma_{\text {red }} \cong \Gamma\left(\mathrm{QB}_{r}\right)^{\mathscr{P}_{r}} . \\
C^{\infty}\left(M_{r}\right) \ni f_{r} \stackrel{\text { quantization }}{\longrightarrow} \tau_{r}\left(f_{r}\right) \text { operator on } \Gamma\left(\mathrm{QB}_{r}\right)^{\mathscr{P}_{r}}
\end{gathered}
$$


The commutativity of this diagram shows that quantization and reduction "commute," i.e., that we can either quantize the reduced phase space or reduce at the quantum level using the BRST symmetry, but that the results will be the same.

Besides these common features, the two applications also differ in some aspects. In the first application (Sect. 5) we assume a proper symplectic action of a connected Lie group $G$ (with Lie algebra $g$ ) on $M_{0}$ and we suppose that $C$ is defined as the zero-level set of an Ad*-equivariant momentum map $J$ for which $0 \in \mathrm{g}^{*}$ is a regular value. With these assumptions $M=M_{0} \times \mathfrak{g} \times \mathrm{g}^{*}$ is a trivial product and (1.2) is an isomorphism. We refer the reader to the existing literature for the proof that (with some additional conditions) (1.4) is indeed a commutative diagram. In this example we are also able to relate $\Gamma_{\text {red }}$ to a quantization $\Gamma\left(\mathrm{QB}_{0}\right)^{\mathscr{P}_{0}}$ of $M_{0}$ :

$$
\Gamma_{\text {red }} \cong\left\{\psi \in \Gamma\left(\mathrm{QB}_{0}\right)^{\mathscr{P}_{0}} \mid \forall v \in \mathrm{g}: i \cdot \tau_{0}\left(J^{*} v\right) \psi=-(1 / 2) \cdot \operatorname{trace}(\operatorname{ad}(v)) \cdot \psi\right\} .
$$

The shift by $(1 / 2) \cdot \operatorname{trace}(\operatorname{ad}(v))$ might not have a direct physical interpretation, but it is a well known shift in the theory of induced representations. It is needed to "construct" a scalar product on $\Gamma_{\text {red }}$.

In the second application (Sect. 6) we consider an oriented regular foliation $\mathscr{D}_{0}$ on a configuration space $Q_{0}$, with the idea that the leaves of this foliation describe gauge equivalent points, i.e. that $Q_{r}=Q_{0} / \mathscr{D}_{0}$ is the reduced configuration space, which we assume to be a smooth manifold. In the phase space $M_{0}=T^{*} Q_{0}$ we define the constraint set $C$ as the annihilator of $\mathscr{D}_{0}$, i.e., $C=\left\{\beta \in T^{*} Q_{0} \mid \forall x \in \mathscr{D}_{0}: \beta(x)=0\right\}$. It turns out that the reduced symplectic manifold $M_{r}=C / \mathscr{D}_{\text {char }}$ is symplectomorphic to $T^{*} Q_{r}$. We stress that we do not assume that $\mathscr{D}_{0}$ is trivial, i.e., we do not assume the existence of $n$ global vector fields on $Q_{0}$ that span $\mathscr{D}_{0}$. On the other hand, the fact that the constraint set $C$ is induced from a foliation on the configuration on $Q_{0}$ is crucial in the construction of the BRST charge on the extended phase space $M$, which is the cotangent bundle of an extended configuration space $Q$ with $\operatorname{dim}(Q)+\operatorname{dim}\left(Q_{r}\right)=2 \operatorname{dim}\left(Q_{0}\right)$. In this second example we do not tackle the question whether (1.2) is injective and/or surjective; however, we prove the commutativity of (1.4). A comparison with a quantization of $T^{*} Q_{0}$ is difficult because we do not assume triviality of $\mathscr{D}_{0}$. If $\mathscr{D}_{0}$ is trivial, a comparison with a quantization of $T^{*} Q_{0}$ would yield a result similar to (1.5) (see also [Tu2]).

\section{Supermanifolds, Frame Bundles and Densities}

We will work with the geometric version of the super/graded manifolds of Kostant. We begin to establish notations and conventions. For more details on supermanifolds, we refer the reader to the existing literature (e.g., [Ko2, Ba2, dW] and references cited therein). Our basic graded commutative algebra $\mathscr{A}$ will be the full exterior algebra of a countably infinite dimensional real vector space, i.e., $\mathscr{A}=\Lambda \mathbf{R}^{\mathbf{N}}=\left(\bigoplus_{k \in N} \Lambda^{2 k} \mathbf{R}^{\mathbf{N}}\right) \oplus\left(\oplus_{k \in N} \Lambda^{2 k+1} \mathbf{R}^{\mathbf{N}}\right)=\mathscr{A}_{0} \oplus \mathscr{A}_{1}$. We will use the function $\varepsilon$ to denote the parity of an object; it will only appear in the exponent of -1 . If $V$ is a module over $\mathscr{A}$, then elements $e_{1}, \ldots, e_{p+q}$ will be called a basis for $V$ if the following two conditions hold:

(i) $V$ is isomorphic to the free $\mathscr{A}$-module spanned by $e_{1}, \ldots, e_{p+q}$,

(ii) $\varepsilon\left(e_{j}\right)=0$ for $j \leqq p, \varepsilon\left(e_{j}\right)=1$ for $j>p$. 
In such 'a' case we will call $(p, q)$ the dimension of $V$. We will only work with $\mathscr{A}$-modules that admit such a basis. By abuse of notation (and when no confusion is possible) we will call such modules (graded) vector spaces and their elements vectors. By a subspace $W$ of $V$ we will mean any $\mathscr{A}$-submodule $W$ of $V$ which is spanned by a subset of a basis of $V$. We will consider two groups of endomorphisms of a graded vector space $V$; in the first place $S G L(V)$ consisting of all even invertible $\mathscr{A}$-linear maps $V \rightarrow V$. With respect to a basis $e_{1}, \ldots, e_{p+q}$ of $V$ any such map is determined by a block matrix $\left(\begin{array}{ll}A & B \\ C & D\end{array}\right)$ of size $(p+q) \times(p+q)$, where $A$ has size $p \times p, D$ size $q \times q$, both $A$ and $D$ are even and invertible, while $B$ and $C$ are odd. We will denote the group of all such matrices by $S G L(p, q)$. We will also consider the subgroup $S G L_{\pi}(p, q) \subset S G L(p, q)$ of matrices with $\operatorname{Det}(D)>0$. The corresponding subgroup of $S G L(V)$ can be interpreted as those endomorphisms that preserve an orientation on the subspace spanned by $e_{p+1}, \ldots, e_{p+q}$.

A supermanifold $Q$ of dimension $(p, q)$ is defined by open sets $U \subset\left(\mathscr{A}_{0}\right)^{p} \times\left(\mathscr{A}_{1}\right)^{q}$ and $C^{\infty}$ transition functions (abuse of notation: $C^{\infty}$ stands here for super smooth functions, i.e., smooth functions in the even coordinates and polynomials in the odd coordinates). Restriction to the even coordinates defines an ordinary manifold $Q_{0}$, called the underlying real manifold of $Q$. The odd-odd part of the Jacobian matrix (i.e. the submatrix $D$, which is even!) defines an ordinary vector bundle $V Q \rightarrow Q_{0}$ with typical fibre $\mathbf{R}^{q}$. Batchelor [Ba1] has shown that there exists a (non-canonical) isomorphism $C^{\infty}(Q) \cong$ sections of $\left.\Lambda V Q \rightarrow Q_{0}\right\}$. If $U$ is a local chart for $Q$ with coordinates $z^{1}, \ldots, z^{p+q}\left(z^{j}\right.$ even for $j \leqq p$, odd otherwise) then the derivations $\partial_{j}:=\partial / \partial z^{j}$ are a basis for the tangent space $T_{m} Q$ at each point $m \in U \subset Q$. We define the cotangent space $T^{*}{ }_{m} Q$ as the left $\mathscr{A}$-linear maps $f: T_{m} Q \rightarrow \mathscr{A} ; k$-forms at $m$ are elements of $\Lambda T^{*}{ }_{m} Q$.

A distribution $\mathscr{D}$ of dimension $\left(p^{\prime}, q^{\prime}\right)$ on $Q$ is a smooth assignment of a subspace $\mathscr{D}_{m} \subset T_{m} Q$ with $\operatorname{dim} \mathscr{D}_{m}=\left(p^{\prime} \leqq p, q^{\prime} \leqq q\right)$; smooth means that locally there exist smooth vector fields $X_{1}, \ldots, X_{p^{\prime}+q^{\prime}}$ on $Q$ such that $X_{j}$ is even for $j \leqq p^{\prime}$, odd otherwise, and such that $\left.X_{1}\right|_{m}, \ldots,\left.X_{p^{\prime}+q^{\prime}}\right|_{m}$ is a basis of $\mathscr{D}_{m}$. Obviously $\mathscr{D}$ can be considered as a vector bundle over $Q$ with structure group $S G L\left(p^{\prime}, q^{\prime}\right)$. We call $\mathscr{D}$ orientable in the odd directions if the structure group can be reduced to $S G L_{\pi}\left(p^{\prime}, q^{\prime}\right)$. Now define $\mathscr{F} \mathscr{D} \rightarrow Q$ as the bundle of all frames (bases) for $\mathscr{D}$, i.e. a principal $S G L\left(p^{\prime}, q^{\prime}\right)$ bundle over $Q$. If $\mathscr{D}$ is orientable in the odd directions, then the choice of an orientation defines $\mathscr{F} \mathscr{D}^{+} \rightarrow Q$ as the bundle of $\mathscr{D}$-frames which are oriented in the odd directions; it is a principal $S G L_{\pi}\left(p^{\prime}, q^{\prime}\right)$ bundle over $Q$. In the sequel we will use the abbreviation OOD for Oriented in the Odd Directions. We will say that the manifold $Q$ is OOD if the full tangent space $T Q$, seen as a distribution in $Q$, is OOD. In the translation to ordinary manifolds, a supermanifold is OOD if and only if the vector bundle $V Q \rightarrow Q_{0}$ is an oriented vector bundle; in the terminology of Shander [Sh] an OOD manifold is called $(0,1)$ semi-oriented.

Now for $d \in \mathbf{R}$ consider the representation $\mathrm{ABer}^{d}$ (Absolute value of the Berezinian, power $d$ ) of $S G L_{\pi}\left(p^{\prime}, q^{\prime}\right)$ on $\mathscr{A}_{0}$ defined by:

$$
\operatorname{ABer}^{d}\left(\left(\begin{array}{ll}
A & B \\
C & D
\end{array}\right)\right)=\left[\left|\operatorname{Det}\left(A-B D^{-1} C\right)\right| \cdot \operatorname{Det}(D)^{-1}\right]^{-d}
$$

If $\mathscr{D}$ is an OOD distribution on $Q$ then we can consider the line bundle $\Delta^{d} \mathscr{D} \rightarrow Q$ associated to $\mathscr{F} \mathscr{D}^{+}$by this representation. Sections of this bundle can be identified 
in the standard way with functions $v$ on $\mathscr{F} \mathscr{D}^{+}$satisfying:

$$
v\left(m ;\left(e_{j}\right) g\right)=\operatorname{ABer}^{d}\left(g^{-1}\right) v\left(m ;\left(e_{j}\right)\right),
$$

or equivalently

$$
v\left(m ;\left(e_{j}\right)\left(\begin{array}{ll}
A & B \\
C & D
\end{array}\right)\right)=\left|\operatorname{Det}\left(A-B D^{-1} C\right)\right|^{d} \cdot \operatorname{Det}(D)^{-d} \cdot v\left(m ;\left(e_{j}\right)\right),
$$

where $g \in S G L_{\pi}\left(p^{\prime}, q^{\prime}\right)$ and $e_{1}, \ldots, e_{p^{\prime}+q^{\prime}}$ an OOD basis for $\mathscr{D}_{m}$. If we consider functions on $\mathscr{F} \mathscr{D}^{+}$with values in $\mathscr{A}$ satisfying (2.2/3), we will call the line bundle $\Delta^{d} \mathscr{D}$ a real line bundle; it is the direct generalization to supermanifolds of the usual notion of a real line bundle. However, we want to use such a line bundle in the construction of the quantum Hilbert space, which should be a Hilbert space over $\mathbf{C}$. Hence, instead of considering functions on $\mathscr{F} \mathscr{D}^{+}$with values in $\mathscr{A}$, we will consider functions on $\mathscr{F} \mathscr{D}^{+}$with values in $\mathscr{A} \otimes \mathbf{C}=\mathscr{A}+i \mathscr{A}$ satisfying $(2.2 / 3)$, and we will call the line bundle $\Delta^{d} \mathscr{D}$ a complex line bundle. The (complex) line bundle $\Delta^{d} \mathscr{D}$ has dimension $(p+2, q+2)$. A partition of unity argument shows that $\Delta^{d} \mathscr{D}$ is trivial, i.e., that there exists an even smooth section $v_{0}$ such that every other section $v$ can be given as $v=f \cdot v_{0}$ for some function $f \in C^{\infty}(Q)$.

If $Q$ were an ordinary manifold and $Y$ a vector field on $Q$ with flow $\varphi_{t}$ preserving $\mathscr{D}$, then there would be a canonical lift of $\varphi_{t}$ to a flow $\hat{\varphi}_{t}$ on $\mathscr{F} \mathscr{D}^{(+)}$ defined by $\hat{\varphi}_{t}\left(m ;\left(e_{j}\right)\right)=\left(\varphi_{t}(m) ;\left(\varphi_{t^{*}} e_{j}\right)\right)$. The infinitesimal generator $\hat{Y}$ of $\hat{\varphi}_{t}$ would be a vector field on $\mathscr{F} \mathscr{D}^{(+)}$, invariant under the $G L\left(p^{\prime}\right)$ action and projecting to $Y$. Although there does not exist a flow for a general vector field on a supermanifold, nevertheless we can define vector fields $\hat{Y}$ with the desired properties.

Lemma 2.4. For each vector field $Y$ on $Q$ preserving $\mathscr{D}$, i.e. $[Y, \mathscr{D}] \subset \mathscr{D}$, there exists a vector field $\hat{Y}$ on $\mathscr{F} \mathscr{D}^{(+)}$with the following properties:

(i) If $q=0$ then $\hat{Y}$ is as before;

(ii) $\pi_{*} \hat{Y}=Y\left(\pi: \mathscr{F} \mathscr{D}^{(+)} \rightarrow Q\right)$;

(iii) $\hat{Y}$ is invariant under the $S G L_{\pi}\left(p^{\prime}, q^{\prime}\right)$ action and

(iv) If $Z$ also preserves $\mathscr{D}$ then $\widehat{[Y, Z]}=[\hat{Y}, \hat{Z}]$.

The vector field $\hat{Y}$ is defined locally by the following procedure. Let $X_{1}, \ldots, X_{p^{\prime}+q^{\prime}}$ be vector fields which span $\mathscr{D}$ on $U \subset Q$ and which are OOD in the case of $\mathscr{F} \mathscr{D}^{+}$, then there exist functions $\lambda$ on $U$ such that $\left[X_{j}, Y\right]=\sum_{k} X_{k} \cdot \lambda^{k}{ }_{j}$. Moreover, the $X_{j}$ identify $\left.\mathscr{F} \mathscr{D}^{(+)}\right|_{U}$ with $U \times S G L_{(\pi)}\left(p^{\prime}, q^{\prime}\right)$ by:

$$
\left(e_{j}\right) \text { basis of } \mathscr{D}_{m} \Leftrightarrow e_{j}=\left.\sum_{k} X_{k}\right|_{m} \cdot a_{j}^{k}, \quad a^{k} \in S G L_{(\pi)}\left(p^{\prime}, q^{\prime}\right) .
$$

In this trivialisation $\hat{Y}$ is given by:

$$
\left.\hat{Y}\right|_{(m, a)}=\left.Y\right|_{m}+\sum_{u j k}(-1)^{\varepsilon(Y)(\varepsilon(j)+\varepsilon(k))} \cdot \lambda^{k}{ }_{u} \cdot a^{u}{ }_{j} \cdot \partial /\left.\partial a^{k}{ }_{j}\right|_{a},
$$

where $\varepsilon(u)$ is shorthand for $\varepsilon\left(X_{u}\right)$. Our claim is that this gives a globally defined vector field on $\mathscr{F} \mathscr{D}^{(+)}$with the stated properties; the proof is a straightforward calculation.

As the last definition of this section we introduce the set $\Omega^{d}(\mathscr{D})$ of smooth sections of $\Delta^{d} \mathscr{D}$ and its subspace $\Omega^{d}{ }_{c}(\mathscr{D})$ of those sections with compact support in $Q_{0}$. Depending on the context we will interpret elements of $\Omega^{d}(\mathscr{D})$ either' as sections 
of $4^{d} \mathscr{D}$ or 'as functions on $\mathscr{F} \mathscr{D}^{+}$; they will be called $d$ - $\mathscr{D}$-densities on $Q$ (or simply $d$-densities if $\mathscr{D}=T Q$ ). The vector fields $\hat{Y}$ of $(2.4 / 6)$ allow us to define a Lie derivative on $\Omega^{d}(\mathscr{D})$ by:

$$
\mathscr{L}(Y) v=\hat{Y} v,
$$

where the left-hand side is in the section mode and the RHS in the function mode. In this way, the map $Y \rightarrow \mathscr{L}(Y)$ from $\mathscr{D}$-preserving vector fields on $Q$ to operators on $\Omega^{d}(\mathscr{D})$ is left $\mathscr{A}$-linear and preserves brackets (N.B. $\Omega^{d}(\mathscr{D})$ has a natural grading, so in defining the bracket of operators one has to allow for signs). Now if $X_{j}$ span $\mathscr{D}$ on $U$ as above and if $v$ is an element of $\Omega^{d}(\mathscr{D})$ then we get a function $f$ on $Q$ defined by $f(m)=v\left(m ;\left(\left.X_{j}\right|_{m}\right)\right)$. If $Y$ is a $\mathscr{D}$-preserving vector field on $Q$ then it is interesting to know the relation between $Y f$ and $\mathscr{L}(Y) v$. The following lemma follows directly from the definition of $\hat{Y}$ and the properties of the ordinary determinant.

Lemma 2.8. $(Y f)(m)=(\mathscr{L}(Y) v)\left(m ;\left(\left.X_{j}\right|_{m}\right)\right)-d \cdot \operatorname{Div}_{(X)}(Y)(m) \cdot f(m)$, where $\operatorname{Div}_{(X)}(Y)$ is given in terms of the functions $\lambda^{k}{ }_{j}$ (i.e. $\left[X_{j}, Y\right]=\sum_{k} X_{k} \cdot \lambda^{k}{ }_{j}$ ) by

$$
\operatorname{Div}_{(X)}(Y)=\sum_{j}(-1)^{\varepsilon(j)} \cdot \lambda^{j}{ }_{j}
$$

The function $\operatorname{Div}_{(X)}$ depends only upon the vector fields $X$ which trivialise $\mathscr{D}$; it is a generalization of the divergence of a vector field on $\mathbf{R}^{n}$.

It has been shown ([Ro, Le]) that if $Q$ is OOD then Berezin integration gives a well defined map $\Omega^{1}{ }_{c}(T Q) \rightarrow \mathbf{R}$ (or $\mathbf{C}$ ), $v \rightarrow \int_{Q} v$. If we now realise that $\Delta^{d} \mathscr{D} \otimes \Delta^{d^{\prime}} \mathscr{D}=\Delta^{d+d^{\prime}} \mathscr{D}$, or correspondingly $\Omega^{d}(\mathscr{D}) \cdot \Omega^{d^{\prime}}(\mathscr{D}) \subset \Omega^{d+d^{\prime}}(\mathscr{D})$ (just the pointwise multiplication of functions on $\left.\mathscr{F} \mathscr{D}^{+}\right)$, then it follows that there exists a supersesquilinear form $\langle\rangle:, \Omega^{1 / 2}{ }_{c}(T Q) \times \Omega^{1 / 2}{ }_{c}(T Q) \rightarrow$ C defined by:

$$
\langle s, t\rangle=\int_{Q} \bar{s} \cdot t
$$

Remark 2.11. Besides the usual (anti-) linear properties, a supersesquilinear form satisfies the relation $\overline{\langle s, t\rangle}=(-1)^{\varepsilon(s) \cdot \varepsilon(t)} \cdot\langle t, s\rangle$. It is this additional sign which makes the scalar product we will obtain by geometric quantization slightly different from the one used by Kostant and Sternberg in [KS].

It is not hard to show that this form is non-degenerate and (in the presence of both even and odd coordinate) indefinite; $\Omega^{1 / 2}(T Q)$ is the natural generalisation of $1 / 2$-densities on ordinary manifolds.

\section{Symplectic Supermanifolds and Geometric Quantization}

A symplectic supermanifold is a supermanifold $M$ with a closed non-degenerate even 2 -form $\omega$. The generalized Darboux theorem tells us that locally there exist coordinates $x^{j}, y_{j}(j=1 \ldots p)$ even and $\vartheta^{j}(j=1 \ldots q)$ odd such that $\omega$ is given by:

$$
\omega=\sum_{j} d y_{j} \wedge d x^{j}+\sum_{j}(1 / 2) \cdot \varepsilon_{j} \cdot d \vartheta^{j} \wedge d \vartheta^{j},
$$

where $\varepsilon_{j}= \pm 1$; the number of positive signs is an invariant of $\omega([\mathrm{Ko} 2, \mathrm{He}])$. It follows that $\operatorname{dim} M=(2 p, q)$ and that there is a third number $s$ determined by $\omega$; it is called its signature and defined as $s=\#$ positive $\varepsilon-$ \# negative $\varepsilon$. Note that 
$\omega$ induces an ordinary symplectic form $\omega_{0}$ on the underlying real manifold $M_{0}$. For $f \in C^{\infty}(M)$ one defines the hamiltonian vector field $\xi(f)$ as usual:

$$
\imath(\xi(f)) \omega+d f=0 .
$$

The resulting Poisson bracket $\{f, g\}=\xi(f) g$ gives $C^{\infty}(M)$ the structure of a superPoisson algebra, such that the assignment $f \rightarrow \xi(f)$ is a morphism of graded Lie algebras. We finish our remarks on symplectic manifolds with the construction of the Liouville form $\varepsilon_{\omega}$ on $M$. For this we need that $M$ is OOD, so that is what we will assume from now on. Since $M_{0}$ itself is oriented by the symplectic form $\omega_{0}$, our assumption implies that $V M$ itself is oriented (and of course oriented as a vector bundle over $M_{0}$ ). If $e_{1}, \ldots, e_{2 p+q}$ is a basis for $T_{m} M$ then $\omega$ defines a matrix $\omega^{k}{ }_{j} \in S G L(2 p, q)$ by:

$$
\omega^{k}{ }_{j}=\imath\left(e_{k}\right) l\left(e_{j}\right) \omega
$$

and it is easy to check that the sign of the superdeterminant of $\omega^{k}$, $\operatorname{sign} \operatorname{Ber}\left(\omega^{k}{ }_{j}\right)$, is independent of the choice of a basis. On an OOD basis $e_{1}, \ldots, e_{2 p+q}, \varepsilon_{\omega} \in \Omega^{1}(T M)$ is defined by:

$$
\varepsilon_{\omega}\left(m ;\left(e_{j}\right)\right)=\left|\operatorname{Ber}\left(\omega^{k}{ }_{j}\right)\right|^{1 / 2} .
$$

Using the local expression (3.1) for $\omega$ and Lemma 2.8 it is not hard to check that for any locally hamiltonian vector field $X$ on $M$, i.e., such that $\mathscr{L}(X) \omega=0$, one has $\mathscr{L}(X) \varepsilon_{\omega}=0$. In particular for all $f \in C^{\infty}(M)$ we have $\mathscr{L}(\xi(f)) \varepsilon_{\omega}=0$.

We will develop geometric quantization for symplectic supermanifolds as a generalisation of standard geometric quantization. As in standard geometric quantization we have often to prove that our constructions and definitions are valid. However, since these proofs are straightforward adaptations of the proofs for the ordinary case, we will only point out the key ingredients needed for the adaptation to the super case. For the proofs in the standard case we refer to the existing literature ([Ko1, So, SW, Sn, Wo, Tu1]). The first step in this program is the prequantization construction, which is carried out in detail by Kostant [Ko2]. We recall briefly the necessary details. There exists a complex line bundle $L$ over $M$ of dimension $(2 p+2, q+2)$ with hermitian structure (,) and compatible connection $\nabla$ such that curvature $(\nabla)=i \cdot(2 \pi)^{-1} \cdot \omega$, if and only if $\omega_{0}$ is integral. The bundle $L$ is derived from a principal $U(1)$ bundle $Y \rightarrow M$ of dimension $(2 p+1, q)$ by the (even) identity representation $e^{i \varphi} \in S G L(2,2)=S G L(\mathscr{A}+i \mathscr{A})$. The choice of a local potential $\alpha$ for $\omega$, i.e., $d \alpha=\omega$, defines a trivialisation of $L$, identifying local sections of $L$ with $\mathscr{A}+i \mathscr{A}$-valued functions on $M$. In such a trivialisation the connection $\nabla$ is given by:

$$
\nabla_{X} s=X s-i \cdot l(X) \alpha \cdot s .
$$

With the applications in mind we will restrict our attention to real polarizations and 1/2-densities, although we will sometimes make brief excursions to complex polarizations and $1 / 2$-forms. A real polarization $\mathscr{P}$ is an OOD distribution on $M$ with the following two properties:

(i) $\mathscr{P}$ is involutive and

(ii) $\mathscr{P}$ is maximal isotropic with respect to $\omega$. 
Note that it follows that the dimension of $\mathscr{P}$ is $\left(p, q^{\prime}\right)$ with $q^{\prime}=(q+|s|) / 2$, and that the even part of $\mathscr{P}$ defines an ordinary (real) polarization on $\left(M_{0}, \omega_{0}\right)$. The superversion of Frobenius' lemma implies that there exist local functions (part of a coordinate system) $z^{1}, \ldots, z^{p+q^{\prime}}$ such that $\mathscr{P}$ is defined by $d z^{1}=\ldots$ $=d z^{p+q^{\prime}}=0$. Condition (ii) then implies that $\mathscr{P}$ is spanned by the hamiltonian vector fields $\xi\left(z^{j}\right)$. We now make the additional assumption that there exists an OOD manifold $Q$ and a submersion $\pi: M \rightarrow Q$ with connected fibres such that $\mathscr{P}=\operatorname{ker}\left(\pi_{*}\right)$. This manifold $Q=M / \mathscr{P}$ is the generalised configuration space for $M$.

The assumption that $\mathscr{P}$ is OOD enables us to define the quantum line bundle $\mathrm{QB} \rightarrow M$ of dimension $(2 p+2, q+2)$ as

$$
\mathrm{QB}=L \otimes_{C} \Delta^{-1 / 2}(\mathscr{P}) .
$$

On $\Delta^{-1 / 2}(\mathscr{P})$ we define a partial connection by the following procedure. Let $X \in \mathscr{P}_{m}$ and $e_{1}, \ldots, e_{p+q^{\prime}}$ an OOD basis of $\mathscr{P}_{m}$, then there exist functions $z^{1}, \ldots, z^{p+q^{\prime}}$ in a neighbourhood of $m$ such that the $\xi\left(z^{j}\right)$ form a basis of $\mathscr{P}$ and such that $e_{j}=\left.\xi\left(z^{j}\right)\right|_{m}$. Now if $v \in \Omega^{-1 / 2}(\mathscr{P})$, we will define $\nabla_{X} v \in \Omega^{-1 / 2}(\mathscr{P})$ by:

$$
\left(\nabla_{X} v\right)\left(m ;\left(e_{j}\right)\right):=\left.X\right|_{m}\left(v\left(\cdot ;\left(\xi\left(z^{j}\right)\right)\right),\right.
$$

where on the RHS we have interpreted $v$ as a function on $\mathscr{F}^{+}$. Standard techniques show that this is a well defined flat connection, although only for vectors in $\mathscr{P}$. Using Lemma (2.8) and the isotropy of $\mathscr{P}$, one can show that (as for ordinary geometric quantization) if $X \in \mathscr{P}$ is a locally hamiltonian vector field, then $\nabla_{X} v=\mathscr{L}(X) v$. The sum connection on $Q B$ defined by:

$$
\nabla_{X}(s \otimes v)=\left(\nabla_{X} s\right) \otimes v+(-1)^{\varepsilon(X) \cdot \varepsilon(s)} \cdot s \otimes \nabla_{X} v
$$

gives us a partial connection on $Q B$. This allows us to define the set $\Gamma(Q B)^{\mathscr{P}}$ of sections $\psi$ of $Q B$ that are covariant constant in the direction of $\mathscr{P}$, i.e.:

$$
\psi \in \Gamma(Q B)^{\mathscr{P}} \Leftrightarrow \forall X \in \mathscr{P}: \nabla_{X} \psi=0
$$

As for ordinary geometric quantization we claim that there is a natural sesquilinear map $():, \Gamma(\mathrm{QB})^{\mathscr{P}} \times \Gamma(\mathrm{QB})^{\mathscr{P}} \rightarrow \Omega^{1}(T Q)$ which associates to two elements of $\Gamma(\mathrm{QB})^{\mathscr{P}}$ a 1 -density on $Q$. Let $(\xi)$ be vectors in $T_{m} M$ such that $\left(\pi_{*} \xi\right)$ is an OOD basis for $T_{\pi(m)} Q$, let $(\zeta)$ be a basis for $\mathscr{P}_{m}$ and $(\xi, \zeta)$ the OOD basis for $T_{m} M$ formed from the vectors $(\xi)$ and $(\zeta)$, then for $\psi_{j}=s_{j} \otimes v_{j} \in \Gamma(\mathrm{QB})^{\mathscr{P}}$, the scalar product $\left(\psi_{1}, \psi_{2}\right)$ is defined by the formula:

$$
\begin{aligned}
&\left(\psi_{1}, \psi_{2}\right)\left(\pi(m) ;\left(\pi_{*} \xi\right)\right) \\
& \quad=(-1)^{\varepsilon\left(s_{2}\right) \cdot \varepsilon\left(v_{1}\right)} \cdot\left(s_{1}, s_{2}\right)(m) \cdot \overline{v_{1}(m ;(\zeta))} \cdot v_{2}(m ;(\zeta)) \cdot \varepsilon_{\omega}(m ;(\zeta, \xi)) .
\end{aligned}
$$

It is not hard to show that this $\left(\psi_{1}, \psi_{2}\right)$ transforms properly as a 1-density on $Q$; the only serious problem is to show that it is independent of the choice of $m$ for fixed $\pi(m)$. This can be done by choosing locally hamiltonian vector fields for $(\zeta)$, using Lemma 2.8 and the fact that the $\psi_{j}$ are covariantly constant in the direction of $\mathscr{P}$.

We continue to mimick ordinary geometric quantization and we define $\Gamma_{c}(\mathrm{QB})^{\mathscr{P}}$ as those $\psi \in \Gamma(\mathrm{QB})^{\mathscr{P}}$ such that $(\psi, \psi) \in \Omega^{1}{ }_{c}(T Q)$, i.e. those $\psi$ for which the supermeasure $(\psi, \psi)$ on $Q$ has compact support in $Q_{0}$. It follows that for 
$\psi, \chi \in \Gamma_{c}(\mathrm{QB})^{\mathscr{P}}$ we have $(\psi, \chi) \in \Omega^{1}{ }_{c}(T Q)$, hence $\Gamma_{c}(\mathrm{QB})^{\mathscr{P}}$ carries a "natural" supersesquilinear form $\langle$,$\rangle given by:$

$$
\langle\psi, \chi\rangle=\int_{Q}(\psi, \chi) \in \mathbf{C} .
$$

Since this form is in general indefinite, we will not try to "complete" $\Gamma_{c}(\mathrm{QB})^{\mathscr{P}}$, the more so because we will find other problems with inner products in the applications. On the other hand, we will consider $\Gamma(\mathrm{QB})^{\mathscr{P}}$ and $\Gamma_{c}(\mathrm{QB})^{\mathscr{P}}$ as "representation" spaces for the Poisson algebra $C^{\infty}(M)$.

As the final step in the geometric quantization program we define operators $\tau(f)$ on $\Gamma(\mathrm{QB})$ for observables $f \in C^{\infty}(M)$ that preserve the polarization $\mathscr{P}$, i.e. that satisfy $[\xi(f), \mathscr{P}] \subset \mathscr{P}$. As for ordinary geometric quantization we define the action of $\tau(f)$ on $\psi=s \otimes v$ by:

$$
\tau(f) \psi=\left(-i \cdot \nabla_{\xi(f)} s+f \cdot s\right) \otimes v-i \cdot(-1)^{\varepsilon(f) \cdot \varepsilon(s)} s \otimes \mathscr{L}(\xi(f)) v .
$$

In view of a previous remark this reduces to $\tau(f) \psi=-i \cdot \nabla_{\xi(f)} \psi+f \cdot \psi$ when $\xi(f) \in \mathscr{P}$. If $f$ and $g$ both preserve $\mathscr{P}$, then one verifies that $[\tau(f), \tau(f)] \equiv \tau(f) \cdot \tau(g)-(-1)^{\varepsilon(f) \cdot \varepsilon(g)} \cdot \tau(g) \cdot \tau(f)=-i \cdot \tau(\{f, g\})$, i.e., we obtain the following proposition.

Proposition 3.13. The map $i \cdot \tau(f)$ is a homomorphism for the $\mathscr{P}$-preserving subalgebra of $C^{\infty}(M)$ to the operators on $\Gamma(\mathrm{QB})$.

We end this section with the assertion that $\tau(f)$ preserves $\Gamma_{(c)}(\mathrm{QB})^{\mathscr{P}}$ (where $\Gamma_{(c)}(\mathrm{QB})^{\mathscr{P}}$ denotes both $\Gamma_{c}(\mathrm{QB})^{\mathscr{P}}$ and $\left.\Gamma(\mathrm{QB})^{\mathscr{P}}\right)$, hence that $\Gamma_{(c)}(\mathrm{QB})^{\mathscr{P}}$ is indeed a representation space for (the $\mathscr{P}$-preserving part of) the Poisson algebra $C^{\infty}(M)$. Especially if $f$ not only preserves $\mathscr{P}$ but satisfies $\xi(f) \in \mathscr{P}$, a condition equivalent to saying that $f$ is the pull-back of a function on the generalized configuration space $Q$, then (3.12) and the remark following it show that $\tau(f)$ on $\Gamma_{(c)}(\mathrm{QB})^{\mathscr{P}}$ is just multiplication by $f$.

\section{A Simple Example}

In this section we will elaborate on a simple example in great detail. For those who know geometric quantization, this example will not contain any surprise, it will only show where additional minus signs appear. Consider $M=T^{*} V$ of dimension $(0,2 n)$ where $V=\left(\mathscr{A}_{1}\right)^{n}$ is the unique supermanifold of dimension $(0, n)$. Now in general, if $V$ is any supermanifold with local coordinates $z^{1}, \ldots, z^{n}$ (even and odd) and (local) associated coordinates $\zeta_{1}, \ldots, \zeta_{n}$ in the fibres of $T^{*} V$, then the canonical 1 -form $\alpha$ on $T^{*} V$ is locally given by:

$$
\alpha=\sum_{j} d z^{j} \cdot \zeta_{j}
$$

We use this to equip our $M=T^{*} V$ with its canonical symplectic form $\omega=d \alpha$. In (global) coordinates $\eta^{1}, \ldots, \eta^{n}$ on $V$ and dual coordinates $\rho_{1}, \ldots, \rho_{n}$ in the fibres, $\omega$ is given by

$$
\omega=-\sum_{j} d \eta^{j} \wedge d \rho_{j}=-\sum_{j} d \rho_{j} \wedge d \eta^{j}
$$


If we change to coordinates $\beta_{j}=2^{-1 / 2} \cdot\left(\eta^{j}+\rho_{j}\right), \beta_{j+n}=2^{-1 / 2} \cdot\left(\eta^{j}-\rho_{j}\right)$, $1 \leqq j \leqq n$, then $\omega$ is given as $\omega=\sum_{j}(1 / 2) \cdot d \beta_{j} \wedge d \beta_{j}-\sum_{j}(1 / 2) \cdot d \beta_{j+n} \wedge d \beta_{j+n}$, which is the standard form as given in (3.1). In the coordinates $(\eta, \rho)$ the hamiltonian vector field of a function $f$ is given by

$$
\xi(f)=(-1)^{\varepsilon(f)+1} \cdot \sum_{j}\left(\partial f / \partial \rho_{j} \cdot \partial / \partial \eta^{j}+\partial f / \partial \eta^{j} \cdot \partial / \partial \rho_{j}\right)
$$

In this case the prequantization line bundle $L$ is trivial: $L=M \times(\mathscr{A}+i \mathscr{A})$ and we can use the section $s_{t}(m)=(m, 1)$ to identify sections $s$ with functions $\varphi$ by $s=\varphi \cdot s_{t}$. Using the potential $\alpha=\sum_{j} d \eta^{j} \cdot \rho_{j}$ for $\omega$, the connection $\nabla$ is given globally by (3.5) (replacing of course $s$ by $\varphi$ ). As for all cotangent bundles, there is a canonical polarization $\mathscr{P}$ : the vertical one spanned by $\partial / \partial \rho_{j}$.

Remark 4.4. If we consider the case $n=1$, one easily verifies that there are only two possible polarizations: the vertical one spanned by $\partial / \partial \rho$ and the horizontal spanned by $\partial / \partial \eta$; even allowing complex coefficients does not change this fact. Hence the natural analogue of a holomorphic polarization does not exist for the odddegrees of freedom. However, for $n>1$ there do exist non-real polarizations in the odd directions.

The generalized configuration space $Q$ is $V$ with its canonical projection $\pi: M=T^{*} V \rightarrow V$. Since we are working with vector spaces, it is obvious that we can satisfy the orientation conditions: we orient $T M$ by declaring the basis $\partial / \partial \eta^{1}, \ldots, \partial / \partial \eta^{n}, \partial / \partial \rho_{1}, \ldots, \partial / \partial \rho_{n}$ to be positive; for $\mathscr{P}$ the basis $\partial / \partial \rho_{1}, \ldots, \partial / \partial \rho_{n}$ is positive and for $T Q \partial / \partial \eta^{1}, \ldots, \partial / \partial \eta^{n}$ is positive. Since the vector fields $\partial / \partial \rho_{j}$ globally span $\mathscr{P}, \mathscr{F} \mathscr{P}^{(+)}$is obviously trivial, providing us with a natural trivialising section $v_{t}$ of $\Delta^{-1 / 2}(\mathscr{P})$ by:

$$
v_{t}\left(\eta, \rho ;\left(\partial / \partial \rho_{j}\right)\right)=1
$$

Hence we can identify sections $v$ of $\Delta^{-1 / 2}(\mathscr{P})$ with functions $\chi$ on $M$ by $v=\chi \cdot v_{t}$ and so we can identify sections $\psi$ of $Q B$ with functions $\varphi \cdot \chi$ on $M$ by $\psi=s \otimes v=\varphi \cdot s_{t} \otimes \chi \cdot v_{t}=(\varphi \cdot \chi) \cdot s_{t} \otimes v_{t}$. From now on we will confound the section $\psi$ with the function $\varphi \cdot \chi$ and write $\psi$ for both. If we finally realise that $\partial / \partial \rho_{j}=\xi\left(\eta^{j}\right)$, then we find for $X \in \mathscr{P}: \nabla_{X}\left(\chi \cdot v_{t}\right)=(X \chi) \cdot v_{t}$ and hence

$$
\nabla_{X} \psi=X \psi-i \cdot \imath(X) \alpha \cdot \psi
$$

Since $l\left(\partial / \partial \rho_{j}\right) \alpha=0$, it follows that $\Gamma(\mathrm{QB})^{\mathscr{P}}$ consists of functions $\psi$ that do not depend on $\rho$, i.e., functions on $V$. Since $M_{0}$ is a single point, all sections are compactly supported on $M_{0}$, i.e., $\Gamma_{c}(\mathrm{QB})^{\mathscr{P}}=\Gamma(\mathrm{QB})^{\mathscr{P}} \cong C^{\infty}(V)$ and we have to determine the ingredients of (3.10) to determine the sesquilinear form (3.11) on $\Gamma(\mathrm{QB})^{\mathscr{P}}$. The only unknown there is the Liouville form $\varepsilon_{\omega}$, which is readily determined by (3.4) as

$$
\varepsilon_{\omega}\left(\eta, \rho ; \partial / \partial \eta^{1}, \ldots, \partial / \partial \eta^{n}, \partial / \partial \rho_{1}, \ldots, \partial / \partial \rho_{n}\right)=1
$$

and we find for $\psi_{j} \cong\left(\psi_{j} \cdot s_{t}\right) \otimes v_{t} \in \Gamma(\mathrm{QB})^{\mathscr{P}}$ :

$$
\left(\psi_{1}, \psi_{2}\right)\left(\eta ;\left(\partial / \partial \eta^{j}\right)\right)=\overline{\psi_{1}(\eta)} \cdot \psi_{2}(\eta) \Leftrightarrow\left(\psi_{1}, \psi_{2}\right)=\overline{\psi_{1}} \cdot \psi_{2} \cdot \operatorname{Vol}_{V}
$$


where $\operatorname{Vol}_{V} \in \Omega^{1}(T V)$ is the standard Berezin volume on $V$ defined by $\operatorname{Vol}_{V}\left(\eta ;\left(\partial / \partial \eta^{j}\right)\right)=1$. It follows that the supersesquilinear form $\langle$,$\rangle on$ $\Gamma(\mathrm{QB})^{\mathscr{P}} \cong C^{\infty}(V)$ is given as ordinary Berezin integration over $V$ of functions on $V$.

We now turn our attention to the operators $\tau(f)$. A short calculation using (4.3) shows that the $\mathscr{P}$-preserving functions $f(\eta, \rho)$ are of the form

$$
f(\eta, \rho)=f_{0}(\eta)+\sum_{j} \rho_{j} \cdot f^{j}(\eta)
$$

Now for general $\mathscr{P}$-preserving vector fields $Y$ on $M$ we have $\mathscr{L}(Y)\left(\chi \cdot v_{t}\right)=$ $(Y \chi) \cdot v_{t}+(-1)^{\varepsilon(Y) \cdot \varepsilon(\chi)} \cdot \chi \cdot \mathscr{L}(Y) v_{t}$. Using Lemma (2.8) and the definition (4.5) of $v_{t}$ it follows that $\mathscr{L}(Y) v_{t}=(-1 / 2) \cdot \operatorname{Div}_{(\partial / \partial \rho)}(Y) \cdot v_{t}$. Combining this with the general formula gives:

$$
\mathscr{L}(Y)\left(\chi \cdot v_{t}\right)=\left((Y \chi)-(1 / 2) \cdot \operatorname{Div}_{(\partial / \partial \rho)}(Y) \cdot \chi\right) \cdot v_{t} .
$$

Using again (4.3) we find that for the $\mathscr{P}$-preserving functions $f(4.9)$ we have

$$
\begin{aligned}
{\left[\partial / \partial \rho_{j}, \xi(f)\right] } & =(-1)^{\varepsilon(f)} \cdot \sum_{k} \partial f^{j} / \partial \eta^{k} \cdot \partial / \partial \rho_{k} \\
& =\sum_{k} \partial / \partial \rho_{k} \cdot \partial f^{j} / \partial \eta^{k} \Rightarrow \operatorname{Div}_{(\partial / \partial \rho)}(\xi(f))=-\sum_{j} \partial f^{j} / \partial \eta^{j} .
\end{aligned}
$$

Combining all the formulas we find for the operator $\tau(f)$ the following expression:

$$
\begin{aligned}
\tau(f) \psi & =-i \cdot \xi(f) \psi+(f-\hat{i}(\xi(f)) \alpha) \cdot \psi+(i / 2) \cdot \operatorname{Div}_{(\partial / \partial \rho)}(\xi(f)) \cdot \psi \\
& =-i \cdot(-1)^{\varepsilon(f)+1} \cdot \sum_{j} f^{j} \cdot \partial \psi / \partial \eta^{j}+\left(f_{0}-(i / 2) \cdot \sum_{j} \partial f^{j} / \partial \eta^{j}\right) \cdot \psi .
\end{aligned}
$$

An elementary calculation (but with great attention for signs) shows that for these operators we have:

$$
(i \cdot \tau(f) \psi, \chi)+(-1)^{\varepsilon(f) \cdot \varepsilon(\psi)} \cdot(\psi, i \cdot \tau(f) \chi)=\sum_{j} \partial\left(f^{j} \cdot \bar{\psi} \cdot \chi\right) / \partial \eta^{j} .
$$

If we now realise that Berezin integration of a term $\partial F / \partial \eta$ always yields zero, then this formula gives us after integration the following identity for the operators $\tau(f)$ :

$$
\langle i \cdot \tau(f) \psi, \chi\rangle+(-1)^{\varepsilon(f) \cdot \varepsilon(\psi)} \cdot\langle\psi, i \cdot \tau(f) \chi\rangle=0,
$$

which tells us that $\tau(f)$ is super-hermitian.

\section{Application I: The BRST Charge for a Free Group Action}

A. The Classical Set-Up. Let $\left(M_{0}, \omega_{0}\right)$ be the phase space (symplectic manifold) of a classical system and let $G$ be a connected Lie group of dimension $n$ acting on $M_{0}$ symplectically and admitting an Ad*-equivariant momentum map $J: M_{0} \rightarrow \mathrm{g}^{*}$ (with $g$ the Lie algebra of $G$ ). We suppose that $0 \in \mathrm{g}^{*}$ is a regular value of $J$, and that the reduced phase space $M_{r}=J^{-1}(0) / G$ is a smooth manifold ([MW]). It follows that the reduced phase space can be described by equations on the original phase space $M_{0}$ and in the end taking equivalence classes (points in $J^{-1}(0)$ related by gauge transformations $g \in G$ ). In this set-up, the Poisson algebra $C^{\infty}\left(M_{r}\right)$ can be 
described as the $G$-invariant elements in the quotient $C^{\infty}(M) / I$, where $I$ is the ideal (in $C^{\infty}(M)$ ) of functions vanishing on $J^{-1}(0)$, i.e., $C^{\infty}\left(M_{r}\right) \cong\left(C^{\infty}(M) / I\right)^{G}$. However, this description of the reduced Poisson algebra is not given by equations on the original Poisson algebra and in the end taking equivalence classes, but rather by equations in equivalence classes. If the group $G$ is compact, an averaging procedure shows an isomorphism $\left(C^{\infty}(M) / I\right)^{G} \cong C^{\infty}(M)^{G} / I^{G}$, where the last description is indeed of the form first equations and then equivalence classes. However, in the general case $C^{\infty}\left(M_{r}\right)$ is not described (in a natural way) by equations on $C^{\infty}\left(M_{r}\right)$ and in the end taking equivalence classes (the reader can find a thorough discussion of the various ways to define "reduced Poisson algebras" and their relationships in [AGJ]). It is at this point that the BRST-symmetry comes in: using it one can describe $C^{\infty}\left(M_{r}\right)$ by equations on a Poisson algebra and in the end taking equivalence classes. In the situation described above, it is not hard to explain how this works; why it works is another question altogether.

One starts by replacing $M_{0}$ by the supermanifold $M=M_{0} \times \mathfrak{g} \times \mathrm{g}^{*}=$ $M_{0} \times T^{*} \mathrm{~g}$, where the coordinates on $\mathfrak{g}$ and $\mathrm{g}^{*}$ have to be seen as odd-coordinates. If $b_{1}, \ldots, b_{n}$ is a basis for $\mathfrak{g}$ we denote by $\eta^{1}, \ldots, \eta^{n}$ the (odd) coordinates on $\mathfrak{g}$ with respect to this basis and by $\rho_{1}, \ldots, \rho_{n}$ the dual (odd) coordinates on $\mathfrak{g}^{*}$. We can equip this supermanifold $M$ with a natural symplectic structure $\omega$ given by:

$$
\omega=\omega_{0}+d \alpha=\omega_{0}-\sum_{j} d \rho_{j} \wedge d \eta^{j}=\omega_{0}-\sum_{j} d \eta^{j} \wedge d \rho_{j},
$$

where $\alpha$ is the canonical 1 -form on $T^{*} g$ (see (4.1)). Now suppose $\left(x^{j}, y_{j}\right)$ are canonical (even) coordinates on $M_{0}$ such that $\omega_{0}=\sum_{j} d y_{j} \wedge d x^{j}$, then the hamiltonian vector fields and the Poisson bracket on $M$ are given by:

$$
\begin{aligned}
\{f, g\}=\xi(f) g= & {\left[\partial f / \partial y_{j} \cdot \partial / \partial x^{j}-\partial f / \partial x^{j} \cdot \partial / \partial y_{j}\right.} \\
& \left.-(-1)^{\varepsilon(f)} \cdot\left(\partial f / \partial \rho_{j} \cdot \partial / \partial \eta^{j}+\partial f / \partial \eta^{j} \cdot \partial / \partial \rho_{j}\right)\right] g .
\end{aligned}
$$

With this bracket $C^{\infty}(M)$ becomes a (super) Poisson algebra, and the equivalence with the usual, more algebraic approach is not hard to indicate. The ordinary vector bundle $V M \rightarrow M_{0}$ is just the trivial bundle $V M=M_{0} \times\left(\mathfrak{g} \oplus \mathrm{g}^{*}\right)$, which tells us that $C^{\infty}(M) \cong C^{\infty}\left(M_{0}\right) \otimes \Lambda\left(\mathfrak{g} \oplus \mathrm{g}^{*}\right)$. Note however that the Poisson bracket (5.2) differs from the Poisson bracket of [KS] by a factor 2 in the odd terms. This factor 2 originates in the conventions of Clifford algebras.

On the symplectic manifold $(M, \omega)$ one then defines two functions: $\Omega$, called the "BRST-charge," and Td, called the "total degree" or "ghost number." They are given by:

$$
\begin{aligned}
\operatorname{Td}(m, \eta, \rho) & =\sum_{j} \eta^{j} \cdot \rho_{j}, \\
\Omega(m, \eta, \rho) & =\sum_{j} J_{j}(m) \cdot \eta^{j}-(1 / 2) \cdot \sum_{u j k} c^{u}{ }_{j k} \cdot \eta^{j} \cdot \eta^{k} \cdot \rho_{u},
\end{aligned}
$$

where $J_{j}=J^{*} b_{j}\left(\Leftrightarrow J_{j}(m)=\left\langle J(m), b_{j}\right\rangle\right)$ and where $c^{u}{ }_{j k}$ are the structure constants of $g$ with respect to the basis $b_{j}$, i.e., $\left[b_{j}, b_{k}\right]=\sum_{u} c^{u}{ }_{j k} \cdot b_{u}$. Note that $\Omega$ and Td are well defined on $M$, independent of the choice of the basis $(b)$ for $\mathfrak{g}$. The important feature of $\Omega$ is that it satisfies $\{\Omega, \Omega\}=0$, which implies that for all $f \in C^{\infty}(M)$ one has $\{\Omega,\{\Omega, f\}\}=0$. Using these two functions one then can show:

$$
C^{\infty}\left(M_{r}\right) \cong\left\{f \in C^{\infty}(M) \mid\{\Omega, f\}=\{\mathrm{Td}, f\}=0\right\} \text { modulo }\left\{\{\Omega, g\} \mid g \in C^{\infty}(M)\right\}
$$


which is exactly the desired form: $C^{\infty}\left(M_{r}\right)$ is given by equations on $C^{\infty}(M)$ and in the end taking equivalence classes. Notice that $\{\mathrm{Td}, f\}=k \cdot f$ if and only if in each additive term of $f$ the number of factors $\eta$ (ghosts) minus the number of factors $\rho$ (anti-ghosts) equals $k$. This should explain the name ghost number for $\mathrm{Td}$; the name total degree stems from the cohomological construction used to prove the isomorphism (5.5). The actual isomorphism is given by the following procedure. Let $f \in C^{\infty}(M)$ be a representative of an equivalence class [ $\left.f\right]$ on the RHS of (5.5). Define $f_{0} \in C^{\infty}\left(M_{0}\right)$ by $f_{0}(m)=f(m, \eta=0, \rho=0)$, then $\{\Omega, f\}=0$ guarantees that $f_{0}$ restricted to $J^{-1}(0)$ is invariant under gauge transformations and hence defines a function $\left[f_{0}\right]$ on $M_{r}$. The map $[f] \rightarrow\left[f_{0}\right]$ then is the isomorphism of $(5.5)$.

Apart from taking equivalence classes, formula (5.5) tells us that functions on $M_{r}$ can be identified with functions on $M$ that are invariant under the hamiltonian vector fields associated to $\Omega$ and Td. Hence it is natural to ask whether these vector fields can be integrated to the symplectic action of a group on $M$. The answer is positive: these vector fields integrate to a symplectic action of the group $S=\left\{\left(\begin{array}{ll}g & 0 \\ \chi & 1\end{array}\right) \mid g>0\right.$ even, $\chi$ odd $\} \subset S G L(1,1)$ on $M$. To avoid cumbersome notations, we will denote the matrix $\left(\begin{array}{ll}g & 0 \\ \chi & 1\end{array}\right) \in S \subset S G L(1,1)$ simply by $(g, \chi)$. If $U \subset \mathbf{R}^{2 k}$ is a local chart for $M_{0}$, then we can identify $T_{m} U$ with $\mathbf{R}^{2 k}$ and hence $\left.\xi_{0}\left(J_{j}\right)\right|_{m}$ becomes an element of $\mathbf{R}^{2 k}$. Here $\xi_{0}\left(J_{j}\right)$ means the hamiltonian vector field of $J_{j} \in C^{\infty}\left(M_{0}\right)$ with respect to $\omega_{0}$. With these preparations, the flows $\psi_{\chi}$ of $\xi(\Omega)$ and $\varphi_{t}$ of $\xi(\mathrm{Td})$ acting on $\left(m, \eta^{j}, \rho_{j}\right) \in U \times \mathfrak{g} \times \mathfrak{g}^{*} \subset M$ are given by:

$$
\begin{gathered}
\varphi_{t}(m, \eta, \rho)=\left(m, e^{t} \cdot \eta, e^{-t} \cdot \rho\right) \\
\psi_{\chi}\left(\begin{array}{c}
m \\
\eta^{u} \\
\rho_{j}
\end{array}\right)=\left(\begin{array}{l}
m+\left.\chi \cdot \sum_{j} \eta^{j} \cdot \xi_{0}\left(J_{j}\right)\right|_{m} \\
\eta^{u}-\chi \cdot(1 / 2) \cdot \sum_{j k} c^{u}{ }_{j k} \cdot \eta^{j} \cdot \eta^{k} \\
\rho_{j}+\chi \cdot\left(J_{j}(m)-\sum_{u k} c^{u}{ }_{j k} \cdot \eta^{k} \cdot \rho_{u}\right)
\end{array}\right)
\end{gathered}
$$

Here $t$ is an even parameter and $\chi$ odd; this reflects the parity of the corresponding vector fields. It is obvious that $\varphi$ satisfies $\varphi_{t} \cdot \varphi_{t^{\prime}}=\varphi_{t+t^{\prime}}$; the fact that $\psi$ also satisfies $\psi_{\chi} \cdot \psi_{\chi^{\prime}}=\psi_{\chi}+\chi^{\prime}$ depends heavily upon the fact that $[\xi(\Omega), \xi(\Omega)]=0$ $(\Leftarrow\{\Omega, \Omega\}=0)$, something which is not valid in general for odd-degree vector fields.

It is easy to verify that $\psi_{\chi} \cdot \varphi_{t}=\varphi_{t} \cdot \psi_{\exp (t) \chi}$, and hence we obtain the symplectic left action $\Phi$ of the group $S$ (with $e^{t}$ replaced by $g$ ) as:

$$
\Phi(g, \chi)\left(\begin{array}{l}
m \\
\eta^{u} \\
\rho_{j}
\end{array}\right)=\left(\begin{array}{l}
m+\left.\chi \cdot \sum_{j} \eta^{j} \cdot \xi_{0}\left(J_{j}\right)\right|_{m} \\
g \cdot\left(\eta^{u}-\chi \cdot(1 / 2) \cdot \sum_{j k} c^{u}{ }_{j k} \cdot \eta^{j} \cdot \eta^{k}\right) \\
g^{-1} \cdot\left(\rho_{j}+\chi \cdot\left(J_{j}(m)-\sum_{u k} c^{u}{ }_{j k} \cdot \eta^{k} \cdot \rho_{u}\right)\right)
\end{array}\right)
$$


Note that the displacement of $m$ is nilpotent, so the action of $S$ on $M$ projects down to $M_{0}$ (the underlying real manifold) as the trivial action. Note also that the action associated to the total degree corresponds to scaling of the ghosts and the anti ghosts. The net result of these computations is that $C^{\infty}\left(M_{r}\right)$ consists of equivalence classes of functions on $M$ that are globally invariant under the action of $S$. This in contrast to the situation on $M_{0}$, where $C^{\infty}\left(M_{r}\right)$ is represented by equivalence classes of functions on $M_{0}$ that are invariant under the action of $G$ only on $J^{-1}(0)$.

B. Quantization. In view of all previous discussions, we have to quantize the symplectic supermanifold $(M, \omega)$ and at least the observables $\Omega$ and Td. The aim of this subsection is to apply the geometric quantization procedure of Sect. 3 to $(M, \omega)$ and the observables $\Omega$ and Td. Because $\Omega$ contains the original constraint functions $J_{j}$ and because we want to compare our results to a quantization of the original symplectic manifold $\left(M_{0}, \omega_{0}\right)$, we assume that (ordinary) geometric quantization produces a nice quantization of $\left(M_{0}, \omega_{0}\right)$ and the $J_{j}$. In our context of real polarizations this means more precisely that we assume the existence of a $G$ invariant polarization $\mathscr{P}_{0}$ for $\left(M_{0}, \omega_{0}\right)$ (with generalised configuration space $\left.Q_{0}=M_{0} / \mathscr{P}_{0}\right)$ such that $\Gamma_{(c)}\left(\mathrm{QB}_{0}\right)^{\mathscr{P}_{0}}$ is different from $\{0\}$ (i.e. no Bohr Sommerfeld conditions). The $G$-invariance of $\mathscr{P}_{0}$ implies that the $J_{j}$ leave $\mathscr{P}_{0}$ invariant and hence that we obtain by Proposition 3.13 a representation $\tau_{0}$ of $\mathfrak{g}$ as hermitian operators on $\Gamma_{c}\left(\mathrm{QB}_{0}\right)^{\mathscr{P}_{0}}$ (actually, it is $i \cdot \tau_{0}$ which is the representation as skew hermitian operators).

With these data we now proceed to quantize the manifold $(M, \omega)$, bearing in mind the example as described in Sect. 4. First of all, there exists a natural polarization $\mathscr{P}$ on $M$ obtained from $\mathscr{P}_{0}$ by adding the vertical polarization in the odd directions:

$$
\left.\mathscr{P}\right|_{(m, \eta, \rho)}=\left.\mathscr{P}_{0}\right|_{(m)} \oplus\left\{\sum_{j} \lambda_{j} \cdot \partial /\left.\partial \rho_{j}\right|_{(\eta, \rho)} \mid \lambda_{j} \in \mathscr{A}\right\},
$$

where we have extended $\mathscr{P}_{0}$ in the obvious way to an $\mathscr{A}$-module. One easily verifies that $\mathscr{P}$ is indeed involutive and maximal isotropic with respect to $\omega$ if $\mathscr{P}_{0}$ is with respect to $\omega_{0}$. If $Q_{0}=M_{0} / \mathscr{P}_{0}$, it follows immediately that $M / \mathscr{P}=Q_{0} \times \mathfrak{g}$, so there exists a generalised configuration space. Moreover, by orienting the odd directions as in Sect. 4, we can satisfy all our orientability assumptions. Before we go on with the line bundles $L, \Delta^{-1 / 2}(\mathscr{P})$ and $\mathrm{QB}$, we introduce a useful splitting of vector fields on $M$ : each vector field $Y$ on $M$ splits uniquely as $Y=Y_{+}+Y_{-}$, where $Y_{+}$is tangent to the fibres of $p_{1}: M=M_{0} \times T^{*} \mathrm{~g} \rightarrow M_{0}$ and where $Y_{-}$is tangent to the fibres of $p_{2}: M \rightarrow T^{*} \mathrm{~g}$, i.e. $Y_{+}$can be seen as a vector field on $M_{0}$ parametrized by $(\eta, \rho) \in T^{*} \mathfrak{g}$ and $Y_{-}$as a vector field on $T^{*} \mathfrak{g}$ parametrized by $m \in M_{0}$.

Now let $L_{0}$ be a prequantum bundle for $\left(M_{0}, \omega_{0}\right)$, then $L=L_{0} \times T^{*} \mathrm{~g}$ is a prequantum bundle for $(M, \omega)$ and it follows naturally that sections of $L$ can be interpreted as $(\eta, \rho)$-parametrized sections of $L_{0}$. A local analysis of the construction for $L_{0}$ and $L$, using the potential $\alpha$ for the odd-part of $\omega$, shows that the connection on $L$ is given by

$$
\nabla_{X} s=\nabla_{X+} s+X_{-} s-i \cdot l\left(X_{-}\right) \alpha \cdot s,
$$

where we interpret $s$ as a section of $L_{0}$ depending on $(\eta, \rho)$ and where $X_{-} s$ is the differentiation of $s$ with respect to these parameters. For the connection on $\Delta^{-1 / 2}(\mathscr{P})$ we can give a similar interpretation. Each $v \in \Omega^{-1 / 2}(\mathscr{P})$, interpreted 
as a function $v: \mathscr{F} \mathscr{P} \rightarrow \mathscr{A}+i \mathscr{A}$, defines in a natural way a function $\hat{v}: \mathscr{F} \mathscr{P}_{0} \rightarrow \mathscr{A}+i \mathscr{A}$ depending on parameters $(\eta, \rho)$ by:

$$
\hat{v}\left(m, \eta, \rho ;\left(e_{j}\right)\right)=v\left(m, \eta, \rho ;\left(e_{j}, \partial / \partial \rho_{k}\right)\right)=v\left(m, \eta, \rho ;\left(e_{j}, \partial / \partial \rho_{k}\right)\right) / v_{t}\left(\eta, \rho ;\left(\partial / \partial \rho_{k}\right)\right),
$$

where $\left(e_{j}\right)$ is a basis for $\mathscr{P}_{0}$. In this way we obtain a bijection between $\Omega^{-1 / 2}(\mathscr{P})$ and $(\eta, \rho)$-parametrized elements of $\Omega^{-1 / 2}\left(\mathscr{P}_{0}\right)$. Another way of stating the same bijection is the equality $v=v_{t} \otimes \hat{v}$, where $v_{t}$ is the trivialisation of $\Delta^{-1 / 2}\left(\left(\partial / \partial \rho_{j}\right)\right)$ defined in (4.5). From now on we will drop the hat and interpret $v \in \Omega^{-1 / 2}(\mathscr{P})$ as an $(\eta, \rho)$-parametrized section of $\Delta^{-1 / 2}\left(\mathscr{P}_{0}\right)$. In this interpretation the partial connection $\nabla$ on $\Delta^{-1 / 2}(\mathscr{P})$ is given as:

$$
\nabla_{X} v=\nabla_{X+} v+X_{-} v
$$

where we have used that $\nabla_{\partial / \partial \rho} v_{t}=0$ (see Sect. 4). If we now consider the quantum bundle $\mathrm{QB}=L \otimes \Delta^{-1 / 2}(\mathscr{P})$, we see that sections of $\mathrm{QB}$ can be interpreted as $(\eta, \rho)$-parametrized sections of $\mathrm{QB}_{0}=L_{0} \otimes \Delta^{-1 / 2}\left(\mathscr{P}_{0}\right)$. Moreover, since for $X \in \mathscr{P}$ we have $\imath\left(X_{-}\right) \alpha=0$, the partial connection $\nabla$ on QB is given by:

$$
\begin{aligned}
\nabla_{X}(s \otimes v) & =\left(\nabla_{X} s\right) \otimes v+(-1)^{\varepsilon(X) \cdot \varepsilon(s)} \cdot s \otimes \nabla_{X} v \\
& =\nabla_{X+}(s \otimes v)+X_{-}(s \otimes v) \Leftrightarrow \nabla_{X} \psi=\nabla_{X+} \psi+X_{-} \psi .
\end{aligned}
$$

It follows immediately that a $\psi$ in $\Gamma(\mathrm{QB})^{\mathscr{P}}$ does not depend on the parameters $\rho$ (just choose $X=\partial / \partial \rho_{j}$ ). If we now realise that the $\eta$ are odd coordinates and hence that smooth functions in $\eta$ are necessarily polynomials of degree $\leqq n$ (i.e. $C^{\infty}(\mathrm{g})$ is finite dimensional), we can deduce from (5.13) that

$$
\Gamma_{(c)}(\mathrm{QB})^{\mathscr{P}}=\Gamma_{(c)}\left(\mathrm{QB}_{0}\right)^{\mathscr{P}_{0}} \otimes C^{\infty}(\mathfrak{g})=C^{\infty}\left(\mathfrak{g}, \Gamma_{(c)}\left(\mathrm{QB}_{0}\right)^{\mathscr{P}_{0}}\right),
$$

i.e. $\Gamma_{(c)}(\mathrm{QB})^{\mathscr{P}}$ consists of $\Gamma_{(c)}\left(\mathrm{QB}_{0}\right)^{\mathscr{P}_{0}}$-valued smooth functions on $\mathfrak{g}$ (as odd degree manifold).

Finally we turn our attention to the sesquilinear form on $\Gamma_{(c)}(\mathrm{QB})^{\mathscr{P}}$ for which, as in Sect. 4, we have to know the Liouville volume $\varepsilon_{\omega}$. Since $M$ is a direct product of two symplectic manifolds, one can write $\varepsilon_{\omega}$ in the obvious way as a tensor product $\varepsilon_{\omega}=\varepsilon_{0} \otimes \varepsilon_{-}$, where $\varepsilon_{0}$ is the Liouville volume of $\left(M_{0}, \omega_{0}\right)$ and $\varepsilon_{-}$the (odd-directions) Liouville volume defined in (4.7). The interpretation of $v \in \Omega^{-1 / 2}(\mathscr{P})$ as a $(\vartheta, \rho)$-parametrized element of $\Omega^{-1 / 2}\left(\mathscr{P}_{0}\right)$ via $(5.11)$ and formula (3.10) gives us for $\psi, \chi \in \Gamma_{(c)}(\mathrm{QB})^{\mathscr{P}}$ :

$$
(\psi, \chi)=(\psi, \chi)_{0} \cdot \mathrm{Vol}_{\mathfrak{g}},
$$

where $(\psi, \chi)_{0}$ is the $(\eta)$-parametrized density on $Q_{0}$ associated to the $(\eta)$-parametrized elements $\psi, \chi \in \Gamma_{(c)}(\mathrm{QB})^{\mathscr{P}}$ and where $\operatorname{Vol}_{\mathrm{g}}$ is the standard Berezin volume on $\mathfrak{g}($ see $(4.8))$. The supersesquilinear form on $\Gamma_{c}(\mathrm{QB})^{\mathscr{P}}$ is then given by integration of $(\psi, \chi)$ over $Q=Q_{0} \times \mathfrak{g}$. In the interpretation $\Gamma_{c}(\mathrm{QB})^{\mathscr{P}}=\Gamma_{c}\left(\mathrm{QB}_{0}\right)^{\mathscr{P}_{0}} \otimes C^{\infty}(\mathrm{g})$ we then have that the supersesquilinear form on $\Gamma_{c}(\mathrm{QB})^{\mathscr{P}}$ equals the tensor product of the (ordinary) sesquilinear form on $\Gamma_{c}\left(\mathrm{QB}_{0}\right)^{\mathscr{P}_{0}}$ and the super-sesquilinear form on $C^{\infty}(\mathfrak{g})$ as obtained in Sect. 4. Note that this representation space is exactly the same as the one used in $[\mathrm{KS}]$ because $C^{\infty}(\mathfrak{g})=\Lambda \mathfrak{g}^{*}$.

Now that we know the spaces $\Gamma_{c}(\mathrm{QB})^{\mathscr{P}}$, we turn our attention to the operators $\tau(f)$ for $\mathscr{P}$-preserving functions $f$. For this we need the Lie derivative $\mathscr{L}(Y) v$, $v \in \Omega^{-1 / 2}(\mathscr{P})$, so it is worthwhile to know how this looks in the interpretation of 
$v$ as parametrized sections of $\Delta^{-1 / 2}\left(\mathscr{P}_{0}\right)$. So let $Y$ be a $\mathscr{P}$-preserving vector field on $M$ and let $X_{k}$ be local vector fields on $M_{0}$ spanning $\mathscr{P}_{0}$, seen as vector fields on $M$ independent of $(\eta, \rho)$. It follows that $\left(X_{k}, \partial / \partial \rho_{j}\right)$ span $\mathscr{P}$ and moreover one can easily show the following properties:

$$
\left[Y_{+}, X_{k}\right],\left[Y_{+}, \partial / \partial \rho_{j}\right] \in \mathscr{P}_{+} \&\left[Y_{-}, X_{k}\right],\left[Y_{-}, \partial / \partial \rho_{j}\right] \in \mathscr{P}_{-},
$$

from which one immediately deduces that $Y_{ \pm}$preserve $\mathscr{P}$, that $Y_{+}$is a $(\eta, \rho)$ parametrized vector field on $M_{0}$ preserving $\mathscr{P}_{0}$, and that $Y_{-}$is a $(m)$-parametrized vector field on $T^{*} \mathfrak{g}$ preserving the vertical polarization. Moreover, these properties also show that $\operatorname{Div}_{(X, \partial / \partial \rho)}\left(Y_{+}\right)=\operatorname{Div}_{(X)}\left(Y_{+}\right)$and $\operatorname{Div}_{(X, \partial / \partial \rho)}\left(Y_{-}\right)=\operatorname{Div}_{(\partial / \partial \rho)}\left(Y_{-}\right)$. If we now apply Lemma 2.8 we find the following equality:

$$
\mathscr{L}(Y) v=\mathscr{L}_{0}\left(Y_{+}\right) v+Y_{-} v-(1 / 2) \cdot \operatorname{Div}_{(\partial / \partial \rho)}\left(Y_{-}\right) \cdot v,
$$

where on the RHS $\mathscr{L}_{0}\left(Y_{+}\right) v$ means the parametrized Lie derivative of $v$ as parametrized section of $\Delta^{-1 / 2}\left(\mathscr{P}_{0}\right)$. Applied to a $\mathscr{P}$-preserving function $f \in C^{\infty}(M)$ we find:

$$
\begin{aligned}
\tau(f)(s \otimes v)= & -i \cdot\left(\nabla_{\xi(f)+} s\right) \otimes v-i \cdot(-1)^{\varepsilon(f) \cdot \varepsilon(s)} \cdot s \otimes \mathscr{L}\left(\xi(f)_{+}\right) v-i \cdot \xi(f)_{-}(s \otimes v) \\
& +\left(f-\imath\left(\xi(f)_{-}\right) \alpha+(i / 2) \cdot \operatorname{Div}_{(\partial / \partial \rho)}\left(\xi(f)_{-}\right)\right) \cdot s \otimes v .
\end{aligned}
$$

Remark 5.19. The above computations which relate the quantization of $M=M_{0} \times T^{*} \mathrm{~g}$ to the quantization of $M_{0}$ carries over nearly word for word to an extension from $M_{0}$ to $M=M_{0} \times T^{*} W$, where $W$ is any (additional) (super) configuration space and where we extend the polarization $\mathscr{P}_{0}$ with the vertical polarization on $T^{*} W$. The only delicate point is the identification of $\Delta^{-1 / 2}(\mathscr{P})$ as parametrized elements of $\Delta^{-1 / 2}\left(\mathscr{P}_{0}\right)$. For this we observe that a partition of unity argument shows that any (super) manifold $W$ admits an everywhere invertible (i.e., trivialising) section $\left(\mathrm{Vol}_{W}\right)^{1 / 2}$ of $\Delta^{1 / 2}(T W)$. This section can be "lifted" to a trivialising section $v_{t}$ of $\Delta^{-1 / 2}\left(\mathscr{P}_{\text {vert }}\right)$ which is covariantly constant in the direction of the vertical polarization $\mathscr{P}_{\text {vert }}$ on $T^{*} W$ (it is the generalisation of (4.5)). With this $v_{t}$ in (5.11), the formulas $(5.12-18)$ are valid, only $\mathrm{Vol}_{\mathfrak{g}}$ in $(5.15)$ has to be replaced by $\mathrm{Vol}_{W}$.

Remark 5.20. We have developed and used geometric quantization for symplectic supermanifolds with real polarizations and (1/2)-densities. The generalization to complex polarizations and (1/2)-forms is rather straightforward, but technical. One can easily imagine that the results of this sub-section (i.e., the density $(\psi, \chi)$ on the generalized configuration space and the operators $\tau(f)$ ) remain valid in the context of complex polarizations and (1/2)-forms, just because geometric quantization of the cotangent bundle of a vector space together with the vertical polarization does not change when we complexify the polarization and/or use (1/2)-forms instead of (1/2)-densities.

With these preparations we are now able to quantize the functions $\Omega$ and $\mathrm{Td}$ (5.3/4). We first compute their hamiltonian vector fields using (5.2):

$$
\begin{aligned}
\xi(\mathrm{Td})= & \sum_{j}\left(\eta^{j} \cdot \partial / \partial \eta^{j}-\rho_{j} \cdot \partial / \partial \rho_{j}\right), \\
\xi(\Omega)= & \sum_{j}\left(\eta^{j} \cdot \xi_{0}\left(J_{j}\right)+J_{j} \cdot \partial / \partial \rho_{j}\right) \\
& -\sum_{u j k} c_{j k}^{u} \cdot\left((1 / 2) \cdot \eta^{j} \cdot \eta^{k} \cdot \partial / \partial \eta^{u}+\eta^{k} \cdot \rho_{u} \cdot \partial / \partial \rho_{j}\right),
\end{aligned}
$$


where $\xi_{0}\left(J_{j}\right)$ is the hamiltonian vector field of $J_{j}$ of $M_{0}$. We observe that they are $\mathscr{P}$-preserving (because the $J_{j}$ are $\mathscr{P}_{0}$-preserving), that $\xi(\Omega)_{+}=\sum_{j} \eta^{j} \cdot \xi_{0}\left(J_{j}\right)$, and that $\xi(\mathrm{Td})_{+}=0$. Careful computations then show that:

$$
\begin{aligned}
\operatorname{Div}_{(\partial / \partial \rho)}\left(\xi(\mathrm{Td})_{-}\right) & =\operatorname{Div}_{(\partial / \partial \rho)}(\xi(\mathrm{Td}))=n, \\
\operatorname{Div}_{(\partial / \partial \rho)}\left(\xi(\Omega)_{-}\right) & =\sum_{j k} c^{j}{ }_{j k} \cdot \eta^{k}=-\sum_{k} \eta^{k} \cdot \operatorname{trace}\left(\operatorname{ad}\left(b_{k}\right)\right),
\end{aligned}
$$

and hence we obtain from (5.18) the operators $\tau(\Omega)$ and $\tau(\mathrm{Td})$ on $\Gamma_{(c)}(\mathrm{QB})^{\mathscr{P}}$ as

$$
\begin{aligned}
\tau(\mathrm{Td}) \psi= & -i \cdot \sum_{j} \eta^{j} \cdot \partial \psi / \partial \eta^{j}+(i \cdot n / 2) \cdot \psi \\
\tau(\Omega) \psi= & \sum_{j} \eta^{j} \cdot\left[\tau_{0}\left(J_{j}\right) \psi-(i / 2) \cdot \operatorname{trace}\left(\operatorname{ad}\left(b_{j}\right)\right) \psi\right] \\
& +(i / 2) \cdot \sum_{u j k} c_{j k}^{u} \cdot \eta^{j} \cdot \eta^{k} \cdot \partial \psi / \partial \eta^{u}
\end{aligned}
$$

where we have interpreted $\psi$ as a $(\eta, \rho)$-parametrized section of QB which is independent of $\rho$ because it is covariantly constant in the direction of $\mathscr{P}$. In terms of the identification $\Gamma_{(c)}(\mathrm{QB})^{\mathscr{P}} \cong \Gamma_{(c)}\left(\mathrm{QB}_{0}\right)^{\mathscr{P}_{0}} \otimes C^{\infty}(\mathfrak{g})$ these operators are given as:

$$
\begin{aligned}
\tau(\mathrm{Td})= & -i \cdot \mathbb{1} \otimes \sum_{j}\left(\eta^{j} \cdot \partial / \partial \eta^{j}-1 / 2\right), \\
\tau(\Omega) \psi= & \sum_{j}\left[\tau_{0}\left(J_{j}\right)-(i / 2) \cdot \operatorname{trace}\left(\operatorname{ad}\left(b_{j}\right)\right)\right] \otimes \eta^{j} \\
& +(i / 2) \cdot \mathbb{1} \otimes \sum_{u j k} c_{j k}^{u} \cdot \eta^{j} \cdot \eta^{k} \cdot \partial / \partial \eta^{u} .
\end{aligned}
$$

Using these expressions and the fact that $\tau_{0}\left(J_{j}\right)$ is supposed to be hermitian, one can prove, as at the end of Sect. 4 , that $\tau(\Omega)$ and $\tau(\mathrm{Td})$ are super-hermitian. As in the classical case, we can integrate the Schrödinger equations $-i \cdot \partial \psi / \partial t=\tau(\mathrm{Td}) \psi$ and $-i \cdot \partial \psi / \partial \chi=\tau(\Omega) \psi$ for these operators to bracket preserving 1-parameter supergroups $\Phi_{t}$ for $\tau(\mathrm{Td})$ and $\Psi_{\chi}$ for $\tau(\Omega)$ on $\Gamma_{(c)}(\mathrm{QB})^{\mathscr{P}}$ :

$$
\begin{aligned}
{\left[\Phi_{t}\left(\psi_{0} \otimes f\right)\right](\eta)=} & \psi_{0} \otimes e^{-n t / 2} \cdot f\left(e^{t} \cdot \eta\right) \\
{\left[\Psi_{\chi}\left(\psi_{0} \otimes f\right)\right](\eta)=} & \psi_{0} \otimes f(\eta)+\chi \cdot\left[\sum _ { j } \left(i \cdot \tau_{0}\left(J_{j}\right) \psi_{0}\right.\right. \\
& \left.+(1 / 2) \cdot \operatorname{trace}\left(\operatorname{ad}\left(b_{j}\right)\right) \psi_{0}\right) \otimes \eta^{j} \cdot f(\eta) \\
& \left.-(1 / 2) \cdot \psi_{0} \otimes \sum_{u j k} c^{u}{ }_{j k} \cdot \eta^{j} \cdot \eta^{k} \cdot \partial f / \partial \eta^{u}\right] \\
\Leftrightarrow & \Psi_{\chi}(\psi)=\psi+\chi \cdot i \cdot \tau(\Omega) \psi,
\end{aligned}
$$

where we interpret $\psi_{0} \otimes f$ as a $\Gamma_{(c)}\left(\mathrm{QB}_{0}\right)^{\mathscr{P}_{0}}$-valued function on $\mathfrak{g}$, with $\psi_{0} \in \Gamma_{(c)}\left(\mathrm{QB}_{0}\right)^{\mathscr{P}_{0}}$ and $f \in C^{\infty}(\mathfrak{g})$. $\Phi_{t}$ obviously is a 1-parameter group; that it preserves brackets follows from the fact that the Berezin volume $\mathrm{Vol}_{\mathfrak{g}}$ transforms with the inverse of the Jacobian. The parameter $\chi$ is odd and hence the fact that $\Psi_{\chi}$ is bracket preserving follows from the fact that $\tau(\Omega)$ is super-hermitian (N.B. $\varepsilon(\tau(\Omega))=1$ and hence $\varepsilon(\chi \cdot \tau(\Omega))=0)$. That $\Psi_{\chi}$ is a 1-parameter group follows from the observation (using Proposition 3.13):

$$
-2 \cdot \tau(\Omega)^{2}=[i \cdot \tau(\Omega), i \cdot \tau(\Omega)]=i \cdot \tau(\{\Omega, \Omega\})=0 .
$$


One verifies the equality $\Phi_{t} \cdot \Psi_{\chi}=\Psi_{\exp (t) \chi} \cdot \Phi_{t}$ and hence we obtain a bracket preserving super-unitary action $U$ of $S$ on $\Gamma_{(c)}(\mathrm{QB})^{\mathscr{P}}$ by:

$$
\begin{aligned}
& U(g, \chi) \psi=\left(\Phi_{-\log (g)} \cdot \Psi_{-\chi}\right)(\psi) \\
& \quad \Leftrightarrow\left[U(g, \chi)\left(\psi_{0} \otimes f\right)\right](\eta)=\psi_{0} \otimes g^{n / 2} \cdot f\left(g^{-1} \cdot \eta\right) \\
& \quad+(1 / 2) \cdot \psi_{0} \otimes g^{n / 2-2} \cdot \chi \cdot \sum_{u j k} c^{u}{ }_{j k} \cdot \eta^{j} \cdot \eta^{k} \cdot \partial f / \partial \eta^{u}\left(g^{-1} \cdot \eta\right) \\
& \quad-\sum_{j}\left[i \cdot \tau_{0}\left(J_{j}\right) \psi_{0}+(1 / 2) \cdot \operatorname{trace}\left(\operatorname{ad}\left(b_{j}\right)\right) \psi_{0}\right] \otimes g^{n / 2-1} \cdot \chi \cdot \eta^{j} \cdot f\left(g^{-1} \cdot \eta\right) .
\end{aligned}
$$

So far we have said nothing about the spectrum of in particular $\tau(\mathrm{Td})$. An elementary calculation shows that the eigenvectors of $\tau(\mathrm{Td})$ are of the form $\psi_{0} \otimes f$, where $f$ is a homogeneous polynomial in $\eta$ of fixed degree $d$; the corresponding eigenvalue is $-i \cdot(d-n / 2)$. One should not be surprised that we have imaginary eigenvalues for super-hermitian operators, because the "scalar product" is not positive definite. On the other hand, if we think of $\mathrm{Td}$ as the ghost number, then the quantum mechanical ghost number operator gh is usually given as $\mathrm{gh}=\sum_{j} \eta^{j} \cdot \partial / \partial \eta^{j}$, which has the same eigenvectors as $\tau(\mathrm{Td})$, but with corresponding eigenvalue $d$. The BRST philosophy now tells us that the reduced Poisson representation space $\Gamma_{\text {red }}$ is obtained as the subspace of the extended Poisson representation space $\Gamma(\mathrm{QB})^{\mathscr{P}}$ defined by $\tau(\Omega) \psi=0$ and $\operatorname{gh}(\psi)=0$, i.e. quantum BRST-charge and quantum ghost number zero. In terms of the super-unitary action $U$ of $S$ on $\Gamma(\mathrm{QB})^{\mathscr{P}}$ this is given as:

$$
\begin{aligned}
\Gamma_{\text {red }} & =\left\{\psi \in \Gamma(\mathrm{QB})^{\mathscr{P}} \mid \tau(\Omega) \psi=0=\sum_{j} \eta^{j} \cdot \partial \psi / \partial \eta^{j} \equiv \operatorname{gh}(\psi)\right\} \\
& =\left\{\psi \in \Gamma(\mathrm{QB})^{\mathscr{P}} \mid \forall s \in S: U(x) \psi=\operatorname{Ber}(s)^{n / 2} \cdot \psi\right\},
\end{aligned}
$$

where $\operatorname{Ber}(s)$ is the superdeterminant of $s$ (i.e. $\operatorname{Ber}(g, \chi)=g$ ). One obtains this result by observing that the infinitesimal generator of $e^{n t / 2} \cdot \Phi_{t}$ is exactly the quantum ghost number.

Let us now investigate how $\Gamma_{\text {red }}$ relates to the original quantization $\Gamma\left(\mathrm{QB}_{0}\right)^{\mathscr{P}}$. In the first place, quantum ghost number zero implies that $\psi \in \Gamma(\mathrm{QB})^{\mathscr{P}}$ is independent of the parameters $\eta$, i.e. $\psi=\psi_{0} \otimes 1$. If we substitute this in $\tau(\Omega) \psi=0$, we obtain:

$$
\begin{aligned}
\Gamma_{\text {red }} & \cong\left\{\psi_{0} \in \Gamma\left(\mathrm{QB}_{0}\right)^{\mathscr{P}_{0}} \mid \forall j: \tau_{0}\left(J_{j}\right) \psi_{0}=(i / 2) \cdot \operatorname{trace}\left(\operatorname{ad}\left(b_{j}\right)\right) \cdot \psi_{0}\right\} \\
& =\left\{\psi_{0} \in \Gamma\left(\mathrm{QB}_{0}\right)^{\mathscr{P}_{0}} \mid \forall v \in \mathfrak{g}: A(v) \psi_{0}+(1 / 2) \cdot \operatorname{trace}(\operatorname{ad}(v)) \cdot \psi_{0}=0\right\},
\end{aligned}
$$

where $A: v=\sum_{j} v^{j} \cdot b_{j} \rightarrow i \cdot \sum_{j} v^{j} \cdot \tau_{0}\left(J_{j}\right)$ is the algebra representation of $g$ as skew . hermitian operators on $\Gamma_{(c)}\left(\mathrm{QB}_{0}\right)^{\mathscr{P}_{0}}$. Now suppose that the algebra representation can be integrated to a (unitary) representation $U_{0}$ of $G$ on $\Gamma_{(c)}\left(\mathrm{QB}_{0}\right)^{\mathscr{P}_{0}}$ then (5.34) translates as

$$
\Gamma_{\text {red }} \cong\left\{\psi_{0} \in \Gamma\left(\mathrm{QB}_{0}\right)^{\mathscr{P}_{0}} \mid \forall g \in G: U_{0}(g) \psi_{0}=\operatorname{Det}(\operatorname{Ad}(g))^{-1 / 2} \cdot \psi_{0}\right\} .
$$

N.B. The function $g \rightarrow \operatorname{Det}(\operatorname{Ad}(g))$ is usually called the modular function on $G$ and $G$ is called unimodular if this function is constant (e.g. all compact groups and groups whose algebra are semi-simple are unimodular). 
Remark 5.36. The appearance of the term $(1 / 2) \cdot \operatorname{trace}(\operatorname{ad}(v))$ (or in other words of the modular function) might seem strange from a physical point of view. However, it is a well known term in the theory of induced representations. Now induced representations and reduction are not exactly inverse operations, but in both cases one is faced with the question of defining a suitable scalar product on the subspace of invariant elements in a representation space (Hilbert space). It turns out that this does not exist unless one shifts to quasi invariant elements by the squareroot of the modular function (the reader is referred to [DEGST] for a discussion connecting reduction, induced representations and geometric quantization in the context of this example).

We now turn our attention to operators on $\Gamma(\mathrm{QB})^{\mathscr{P}}$, and we note that, according to Sect. 4 formula (4.9), $\mathscr{P}$-preserving functions are at most linear in the coordinates $\rho$. (This is a common feature of geometric quantization using the vertical polarization: quantizable observables are linear in momenta.) Since we are especially interested in functions on $M$ associated to the reduced Poisson algebra $C^{\infty}\left(M_{r}\right)$ of (5.5), we first note that $C^{\infty}\left(M_{r}\right)$ consists of equivalence classes. Hence if we want to associate to such an equivalence class an operator on $\Gamma_{\text {red }}$, we have to check that (i) a representative leaves $\Gamma_{\text {red }}$ invariant and (ii) equivalent functions induce the same operator on $\Gamma_{\text {red }}$. We start with (ii): two functions are equivalent if they differ by a function $f$ of the form $f=\{\Omega, g\}$. Such a function automatically satisfies the condition $\{\Omega, f\}=0$, and it satisfies the condition $\{\mathrm{Td}, f\}=0$ if and only if $\{\mathrm{Td}, g\}=-g$. We start focussing attention on the quantization of $g$. The function $g$ is quantizable only if it is linear in $\rho$. This condition together with the condition $\{\mathrm{Td}, g\}=-g$ implies that $g(m, \eta, \rho)=\sum_{j} g^{j}(m) \cdot \rho_{j}$. A further analysis of the condition that $g$ preserves $\mathscr{P}$ shows that the $g^{j}$ must satisfy $\xi_{0}\left(g^{j}\right) \in \mathscr{P}_{0}$. Substituting this in (5.18) and using the remark at the end of Sect. 3 we find $\tau(g)=-i \cdot \sum_{j} g^{j} \cdot \partial / \partial \eta^{j}$, which implies that $\tau(g)$ induces the zero operator on $\Gamma_{\text {red }}$. Finally we infer from Proposition 3.13 that $\tau(\{\Omega, g\})$ induces the zero operator on $\Gamma_{\text {red }}$, "proving" that equivalent functions induce the same operator on $\Gamma_{\text {red }}$. (We put quotes around proving, because we did not prove anything: we did not, and cannot, show for all functions $f$ of the form $f=\{\Omega, g\}$ that $\tau(f)$ induces the zero operator on $\Gamma_{\text {red }}$.)

Next we turn our attention to representations $f$ for $C^{\infty}\left(M_{r}\right)$ for which we want to show that their quantization preserves $\Gamma_{\text {red }}$. Quantizable and representing an element in $C^{\infty}\left(M_{r}\right)$ means in particular at most linear in $\rho$ and $\{\mathrm{Td}, f\}=0$, implying in turn that $f$ must be of the form

$$
f(m, \eta, \rho)=f_{0}(m)+\sum_{j k} f_{j}^{k}(m) \cdot \eta^{j} \cdot \rho_{k}
$$

Instead of analysing in full detail the consequences of the remaining conditions $\{\Omega, f\}=0$ and $f \mathscr{P}$-preserving, we only consider some special cases. If the representative function $f$ does not depend on $\eta$ and $\rho$, i.e., $f_{j}{ }^{k} \equiv 0$, the conditions $\{\Omega, f\}=0$ and $f \mathscr{P}$-preserving imply that $f_{0}$ must be a globally $G$-invariant, $\mathscr{P}_{0}$-preserving function on $M_{0}$. Finally (5.18) then shows that $\tau(f)=\tau_{0}\left(f_{0}\right) \otimes \mathbb{1}$. The $G$-invariance of $f_{0}$ implies that $\left\{J_{j}, f_{0}\right\}=0$ and hence Proposition 3.13 shows that $\tau(f)$ leaves $\Gamma_{\text {red }}$ invariant.

To find representatives of the form (5.37) for which the $f_{j}{ }^{k}$ are not identically zero, we note that the constraint functions $J_{j}$ induce the zero function on $M_{r}$. In general the functions $J_{j}$ are not $G$-invariant (unless $G$ is abelian), i.e., on their own 
they do not form a representative for the zero function on $M_{r}$. However, they admit the following representatives $f_{j}$ on $M$ (see the discussion following formula (5.5)):

$$
f_{j}(m, \eta, \rho)=J_{j}(m)-\sum_{u k} c^{u}{ }_{j k} \cdot \eta^{k} \cdot \rho_{u} .
$$

We leave it to the reader to check that these $f_{j}$ actually satisfy $\left\{\Omega, f_{j}\right\}=0$ and are $\mathscr{P}$-preserving. Since they are of the general form (5.37) they are quantizable; formula (5.18) then gives us for the operators:

$$
\tau\left(f_{j}\right)=\tau_{0}\left(J_{j}\right)-(i / 2) \cdot \operatorname{trace}\left(\operatorname{ad}\left(b_{j}\right)\right)+i \cdot \sum_{u k} c_{j k}^{u} \cdot \eta^{k} \cdot \partial / \partial \eta^{u} .
$$

In view of its definition (5.34) the $\tau\left(f_{j}\right)$ induce, as expected, the zero operators on $\Gamma_{\text {red. }}$.

We can summarise the above discussion by saying that if we stay within the (very restricted) domain of $\mathscr{P}$-preserving functions, then $\Gamma_{\text {red }}$ is a representation space for the reduced Poisson algebra $C^{\infty}\left(M_{r}\right)$ as given in (5.5). However, there is another representation space $\Gamma_{r}$ for $C^{\infty}\left(M_{r}\right)$ : the one obtained by geometric quantization of $M_{r}$ itself. The crucial question obviously is: what is the relation between these two representations $\Gamma_{\text {red }}$ and $\Gamma_{r}$ ? The answer one would like to have is: "these two representations are isomorphic," i.e., there exists an isomorphism between $\Gamma_{r}$ and $\Gamma_{\text {red }}$ that intertwines the quantization of polarization preserving operators. Using additional hypotheses one can indeed prove this result: in [GS] it has been shown using compact groups and Kähler polarizations, in [Go] and [DEGST] it has been shown using compatible real polarizations. We thus may conclude that, under one of these hypotheses, the quantization of reduced systems via quantization of an extended system, using the BRST philosophy, provides us with a "correct" quantization of such a reduced system. It is still an open question what the necessary conditions are under which $\Gamma_{\text {red }}$ and $\Gamma_{r}$ are isomorphic representations spaces.

\section{Application II: The BRST-Charge for a Foliation}

$A$. The Classical Set-Up. Consider a configuration space $Q_{0}$ of dimension $d$ with a regular foliation $\mathscr{D}_{0}$ of rank $n<d$ and suppose we are in a nice situation in which the set of leaves $Q_{r}=Q_{0} / \mathscr{D}_{0}$ is a manifold and such that $\pi: Q_{0} \rightarrow Q_{r}$ is a submersion. We think of points on a leaf as gauge equivalent points in $Q_{0}$ and we call $Q_{r}$ the reduced configuration space. The associated phase spaces are their cotangent bundles equipped with their canonical symplectic forms: $\left(T^{*} Q_{0}, d \alpha_{0}\right)$ and $\left(T^{*} Q_{r}, d \alpha_{r}\right)$, where $\alpha_{0}$ and $\alpha_{r}$ are the respective canonical 1-forms (4.1). One can obtain $T^{*} Q_{r}$ by symplectic reduction from $T^{*} Q_{0}$ in the following way. Let $X$ be any vector field on $Q_{0}$, then we associate to $X$ a function $J_{X}: T^{*} Q_{0} \rightarrow \mathbf{R}$ by

$$
J_{X}(\beta)=\beta\left(\left.X\right|_{q}\right), \quad \beta \in T_{q}^{*} Q_{0} .
$$

We then define a constraint set $C=\left\{\beta \in T^{*} Q_{0} \mid \forall X \in \mathscr{D}_{0}: J_{X}(\beta)=0\right\}$ and one notes the equalities

$$
C=\mathscr{D}_{0}{ }^{\perp} \equiv\left\{\beta \in T^{*} Q_{0} \mid \forall X \in \mathscr{D}_{0}: \beta(X)=0\right\}=\pi^{*}\left(T^{*} Q_{r}\right),
$$


where $\pi^{*}\left(T^{*} Q_{r}\right)$ is the pull-back bundle over $Q_{0}$ by $\pi$. We then invoke Frobenius' lemma to obtain local coordinates $q^{1}, \ldots, q^{d}$ on $Q_{0}$ such that $\mathscr{D}_{0}$ is spanned by $\partial / \partial q^{1}, \ldots, \partial / \partial q^{n}$. Since $J_{\partial / \partial q^{j}}=p_{j}$, where the $p_{j}$ are the momentum coordinates in $T^{*} Q_{0}$ associated to the coordinates $q^{j}$, we see that $C=\mathscr{D}_{0}{ }^{\perp}$ is given by the equations $p_{1}=\ldots=p_{n}=0$. It follows that the push-forward $\pi_{*}: \mathscr{D}_{0}{ }^{\perp} \rightarrow T^{*} Q_{r}$ is given by $\left(q^{1}, \ldots, q^{d}, p_{n+1}, \ldots, p_{d}\right) \rightarrow\left(q^{n+1}, \ldots, q^{d}, p_{n+1}, \ldots, p_{d}\right)$, from which we deduce that $\left(\pi_{*}\right)^{*} \alpha_{r}=\left.\alpha_{0}\right|_{C}$. Hence $\pi_{*}: C \rightarrow T^{*} Q_{r}$ is exactly the symplectic reduction of $C$ by its characteristic foliation $\operatorname{ker}\left(\left.d \alpha_{0}\right|_{C}\right)$. We conclude that the reduced phase space associated to the system in $T^{*} Q_{0}$ described by the constraints $J_{X}$, $X \in \mathscr{D}_{0}$ is exactly the cotangent bundle $T^{*} Q_{r}$. Such a situation has been studied by many authors (e.g. [Ku, MP]). What we intend to do now is to make a construction analogous to the construction in Sect. 5 (see also [MP]), defining a BRSTcharge and a total degree on a symplectic supermanifold, constructing the analogue of (5.5) and finally performing geometric quantization. At the end we will discuss the relation with (geometric) quantization of the reduced phase space $T^{*} Q_{r}$.

The essential idea of our construction is the following: $\mathscr{D}_{0}$ is a sub-vector bundle of $T Q_{0}$ over $Q_{0}$ which we will view as a supermanifold $Q$ in which the fibres of $\mathscr{D}_{0} \rightarrow Q_{0}$ are considered as the odd-degrees of freedom, i.e., $\mathscr{D}_{0}=V Q$ in the language of Sect. 2. We can also describe the supermanifold $Q$ in a more down to earth way. Let $U \subset \mathbf{R}^{d}$ be a local chart of $Q_{0}$ with coordinates $q^{j}$, and let $X_{1}, \ldots, X_{n}$ be independent vector fields on $U$ which span $\mathscr{D}_{0}$. To each such couple we associate a chart $(U, X)=U \times\left(\mathscr{A}_{1}\right)^{n} \subset\left(\mathscr{A}_{0}\right)^{d} \times\left(\mathscr{A}_{1}\right)^{n}$ (extend $U$ in the obvious way to the nilpotent parts) with coordinates $\left(q^{j}, \eta^{k}\right)$. If $\left(U^{\prime}, X^{\prime}\right)$ with coordinates $\left(q^{\prime j}, \eta^{\prime k}\right)$ is another couple with $U \cap U^{\prime} \neq \emptyset$, there exists a coordinate transformation $q^{\prime j}=q^{j}(q)$ and there exist functions $\lambda^{k}{ }_{j}$ on $U \cap U^{\prime}$ such that $X_{j}=\sum_{k} X^{\prime k} \cdot \lambda^{k}{ }_{j}$. With these ingredients we define a coordinate transformation $\left(q^{j}, \eta^{k}\right) \rightarrow\left(q^{\prime j}, \eta^{\prime k}\right)$ by:

$$
q^{\prime j}=q^{j}(q) \quad \eta^{\prime j}=\sum_{k} \lambda^{j}{ }_{k}(q) \cdot \eta^{k}
$$

The collection of all such couples $(U, X)$ with the transition functions (6.3) defines an atlas for the supermanifold $Q$ of dimension $(d, n)$. We then define the extended symplectic supermanifold $M$ as $M=\left(T^{*} Q, d \alpha\right)$; it is the equivalent of the extended phase space as defined in Sect. 5A. If $(q, \eta)$ are local coordinates for $Q$ with associated momenta $(p, \rho)$, and if $\left(q^{\prime}, \eta^{\prime}\right)$ is another set of local coordinates for $Q$ with their momenta $\left(p^{\prime}, \rho^{\prime}\right)$, the coordinate transformation in $T^{*} Q$ between $(q, \eta, p, \rho)$ and $\left(q^{\prime}, \eta^{\prime}, p^{\prime}, \rho^{\prime}\right)$ is given by $(6.3)$ and:

$$
\rho_{j}=\sum_{k} \rho_{k}^{\prime} \cdot \lambda^{k}{ }_{j}(q) \quad p_{j}=\sum_{k} p_{k}^{\prime} \cdot \partial{q^{\prime}}^{\prime} / \partial q^{j}-\sum_{u k} \rho_{u}^{\prime} \cdot \partial \lambda^{u}{ }_{k} / \partial q^{j} \cdot \eta^{k}
$$

In these coordinates the Poisson bracket is given by formula (5.2) (replacing $x$ by $q$ and $y$ by $p$ ).

As in Sect. 5 we can define two global functions Td ("total degree" or "ghost number") and $\Omega$ ("BRST charge") on $T^{*} Q$. Let $(U, X)$ be a local coordinate chart for $Q$ with coordinates $(q, \eta)$ and associated momentum coordinates $(p, \rho)$ in $T^{*} Q$, 
then there exist functions $c^{u}{ }_{j k}$ on $U$ such that $\left[X_{j}, X_{k}\right]=\sum_{u} c^{u}{ }_{j k} \cdot X_{u}$ just because $\mathscr{D}_{0}$ is involutive. In these local coordinates $\mathrm{Td}$ and $\Omega$ are given by:

$$
\begin{aligned}
\operatorname{Td}(q, \eta, p, \rho) & =\sum_{j} \eta^{j} \cdot \rho_{j}, \\
\Omega(q, \eta, p, \rho) & =\sum_{j} J_{X j}(q, p) \cdot \eta^{j}-(1 / 2) \cdot \sum_{u j k} c_{j k}^{u}(q) \cdot \eta^{j} \cdot \eta^{k} \cdot \rho_{u},
\end{aligned}
$$

and an elementary but tedious calculation using the formulas (6.1), (6.3) and (6.4) shows that these local expressions indeed define global functions on $T^{*} Q$. Now we note that by Frobenius' lemma we can always find local coordinates $q$ on $Q_{0}$ such that $\mathscr{D}_{0}$ is spanned by $\partial / \partial q^{1}, \ldots, \partial / \partial q^{n}$. Stated differently, each point in the super-manifold $Q$ admits a local chart $(U, X)$ for which $\left[X_{i}, X_{j}\right]=0$, just by choosing $X_{i}=\partial / \partial q^{i}$ on a local coordinate chart $U$ for $Q_{0}$ given by Frobenius' lemma. It follows that there exist everywhere on $T^{*} Q$ local coordinates in which $\Omega$ is given by $\Omega=\sum_{j} p_{j} \cdot \eta^{j}$. These coordinates on $T^{*} Q$, which we will call adapted coordinates, will be useful in proving global statements that can be verified locally. N.B. Our adapted coordinates are a very special case of what is called by Henneaux the local abelianization of constraints.

Proposition 6.7. With the above definitions we have:

(i) $\{\mathrm{Td}, f\}=k \cdot f$ if and only if in each additive term of $f$ in a local expression the number of factors $\eta$ (ghosts) minus the number of factors $\rho$ (anti-ghosts) equals $k$, and (ii) $\{\Omega, \Omega\}=0$.

Proof. The proof of (i) is an elementary calculation in any local chart of $T^{*} Q$ and left to the reader. The proof of (ii) in an arbitrary local chart for $T^{*} Q$ is a rather long calculation in which the Jacobi identity for vector fields plays an essential role. However, such a lengthy calculation can be avoided entirely by using adapted coordinates (which exist everywhere!): in adapted coordinates we have $\Omega=\sum_{j} p_{j} \cdot \eta^{j}$ and hence, using formula (5.2), it follows immediately that $\{\Omega, \Omega\}=0$.

Remark 6.8. It may come as a surprise that $\{\Omega, \Omega\}=0$ when the $c^{u}{ }_{j k}$ in (6.6) are functions rather than constants. However, in view of the local abelianization theorem, the miracle is not so much that $\{\Omega, \Omega\}=0$ as the fact that the local expressions (6.6) glue together to define a global function $\Omega$. This is entirely due to our special situation, i.e., the fact that the constraint functions are obtained from a foliation defined on a configuration space.

Remark 6.9. In the proof of Theorem 6.7(i) the reader has shown that the difference between the number of factors $\eta$ and factors $\rho$ in a function on $T^{*} Q$ is invariant under coordinate changes. However, the number of factors $\eta$ separately is not invariant under coordinate changes.

Using Td and $\Omega$ we can define the BRST-cohomology in dimension $k$ as:

$$
\begin{aligned}
H_{\text {BRST }}^{k}=\left\{f \in C^{\infty}\left(T^{*} Q\right) \mid\{\mathrm{Td}, f\}=k \cdot f \&\{\Omega, f\}=0\right\} \text { modulo } \\
\\
\left\{f=\{\Omega, g\} \mid g \in C^{\infty}\left(T^{*} Q\right) \&\{\mathrm{Td}, g\}=(k-1) \cdot g\right\} .
\end{aligned}
$$


If $\left\{\mathrm{Td}, f_{j}\right\}=k_{j} \cdot f_{j}$ then $\left.\left\{\mathrm{Td},\left\{f_{1}, f_{2}\right)\right\}\right\}=\left(k_{1}+k_{2}\right) \cdot\left\{f_{1}, f_{2}\right\}$. Using this one can easily prove that the Poisson bracket on $C^{\infty}\left(T^{*} Q\right)$ induces a Poisson bracket on $H^{0}{ }_{\text {BRST }}$ : if $f_{j}$ represent classes $\left[f_{j}\right]$ in $H^{0}{ }_{\text {BRST }}$, then $\left\{\left[f_{1}\right],\left[f_{2}\right]\right\}=\left[\left\{f_{1}, f_{2}\right\}\right]$ (to prove that it is well defined, one uses the Jacobi identity for the Poisson bracket and $\{\Omega, \Omega\}=\left\{\Omega, f_{j}\right\}=0$ ). Moreover, the map described in Sect. 5 following formula (5.5) provides us with a well defined map $H_{\text {BRST }} \rightarrow C^{\infty}\left(T^{*} Q_{r}\right)$ and one can show that it is a morphism of Poisson algebras. At present the author is unable to prove that this morphism is an isomorphism.

Remark 6.11. Our approach to the extended phase space and the BRST charge differs from the (much more general) approach in [FHST] in the sense that they force the extended phase space to be a trivial product of the added (anti-)ghostdirections with the original phase space, whereas we allow twists. They then can prove an isomorphism between $C^{\infty}\left(M_{r}\right)$ and (their) zero dimensional BRST cohomology. The price one has to pay is that one might need more ghosts (possibly infinitely many more) than in our approach.

We continue to mimick Sect. 5 and note that we can integrate the hamiltonian vector fields $\xi(\mathrm{Td})$ and $\xi(\Omega)$ associated to the total degree and the BRST-charge to a symplectic action of the supergroup $S$ on $T^{*} Q$. The local expressions for this action are exactly the same as the ones given in (5.6-8), except that the vector fields $\xi_{0}\left(J_{j}\right)$ have to be replaced by $\xi_{0}\left(J_{X_{j}}\right)$, i.e. the components of the momentum map have to be replaced by the generating constraint functions $J_{X}$, associated to the local basis $X_{1}, \ldots, X_{n}$ for $\mathscr{D}_{0}$ (and of course the structure constants $c^{u}{ }_{j k}$ become functions on $Q_{0}$ ). In the next subsection we will be more explicit about this action and show that there is an action of $S$ on $Q$ such that the action described here is the canonical cotangent bundle lift of that action to $T^{*} Q$.

B. Quantization. We now proceed in this subsection to apply geometric quantization to the symplectic supermanifold $T^{*} Q$. We choose the obvious vertical polarization $\mathscr{D}$ spanned by the vector fields $\partial / \partial p_{j}, \partial / \partial \rho_{k}$ or equivalently by the (hamiltonian) vector fields $\xi\left(q^{j}\right), \xi\left(\eta^{k}\right)$. The "generalized" configuration space is $Q$ itself and we only have to worry about the orientability assumptions, which are satisfied if and only if the foliation $\mathscr{D}_{0}$ is an orientable foliation, i.e. $\mathscr{D}_{0}$ is an orientable vector bundle over $Q_{0}$. As prequantization line bundle $L$ we use the trivial one (this choice is always possible for cotangent bundles), which allows us to identify sections of $L$ with $(\mathscr{A}+i \mathscr{A})$-valued functions on $T^{*} Q$ and hence sections of QB with sections of $\Delta^{-1 / 2}(\mathscr{P})$. Now, to make a long story short, one can identify $\Gamma_{(c)}(\mathrm{QB})^{\mathscr{P}}$ in a canonical way with $\Omega^{1 / 2}{ }_{(c)}(T Q)$. In local coordinates $\left(z^{1}, \ldots, z^{d+n}\right) \equiv\left(q^{1}, \ldots, q^{d}, \eta^{1}, \ldots, \eta^{n}\right)$ on $Q$ with associated momenta $\left(\zeta_{1}, \ldots, \zeta_{d+n}\right) \equiv\left(p_{1}, \ldots, p_{d}, \rho_{1}, \ldots, \rho_{n}\right)$ the element $\psi \in \Gamma_{(c)}(\mathrm{QB})^{\mathscr{P}}$ is identified with $\varphi \in \Omega_{(c)}^{1 / 2}(T Q)$ by

$$
\varphi\left(z ;\left(\partial / \partial z^{j}\right)\right)=\psi\left(z, \zeta ;\left(\xi\left(z^{j}\right)\right)\right)
$$

N.B. The fact that $\psi$ is in $\Gamma(\mathrm{QB})^{\mathscr{P}}$ implies that the RHS of (6.12) is actually independent of $\zeta$. As in Sect. 4, functions which preserve $\mathscr{P}$ are at most linear in momenta. This means more precisely that $f \in C^{\infty}\left(T^{*} Q\right)$ preserves $\mathscr{P}$ if and only if there exist a function $f_{Q} \in C^{\infty}(Q)$ and a vector field $Y$ on $Q$ such that $f=f_{Q}+J_{Y}$, 
where $J_{Y}$ is defined as in (6.1). The translation of the operators $\tau(f)$ to the identification of $\Gamma(\mathrm{QB})^{\mathscr{P}}$ with $\Omega^{1 / 2}(T Q)$ gives us

$$
\tau(f) \varphi=f_{Q} \cdot \varphi-i \cdot \mathscr{L}(Y) \varphi .
$$

A glance at formula (6.5/6) shows that both $\mathrm{Td}$ and $\Omega$ are of the specified form: $f_{Q}=0$ while the vector fields $Y$ are given locally as:

$$
\begin{aligned}
\left.Y_{T D}\right|_{(q, \eta)} & =\sum_{j} \eta^{j} \cdot \partial / \partial \eta^{j}, \\
\left.Y_{\Omega}\right|_{(q, \eta)} & =\left.\sum_{j} \eta^{j} \cdot X_{j}\right|_{q}-(1 / 2) \cdot \sum_{u j k} c_{j k}^{u}(q) \cdot \eta^{j} \cdot \eta^{k} \cdot \partial / \partial \eta^{u} .
\end{aligned}
$$

With these and (6.13) we know the operators $\tau(\mathrm{Td})$ and $\tau(\Omega)$ and, as in Sect. 5, we can integrate the associated Schrödinger equations to a (super-unitary) action of $S$ on $\Gamma_{(c)}(\mathrm{QB})^{\mathscr{P}} \cong \Omega^{1 / 2}{ }_{(c)}(T Q)$. However, in this case we can do slightly better: the vector fields $Y_{T D}$ and $Y_{\Omega}$ have "the same" properties as $\xi(\mathrm{Td})$ and $\xi(\Omega)$, especially $\left[Y_{\Omega}, Y_{\Omega}\right]=0$, and "hence" we can integrate these vector fields to an action of $S$ on $Q$; the action of $S$ on $\Omega^{1 / 2}{ }_{(c)}(T Q)$ is then just the pull-back of $(1 / 2)$-densities. Proceeding as in Sect. 5A we obtain the $S$ action $\Phi$ in a local coordinate chart $(U, X)$ for $Q$ as

$$
\Phi(g, \chi)(q, \eta)=\left(q+\left.\chi \cdot \sum_{j} \eta^{j} \cdot X_{j}\right|_{q}, g \cdot\left(\eta^{u}-\chi \cdot(1 / 2) \cdot \sum_{j k} c^{u}{ }_{j k}(q) \cdot \eta^{j} \cdot \eta^{k}\right)\right)
$$

The interpretation of this action is the same as in Sect. 5A: the $g$-part is scaling of the ghost directions; the $\chi$-part moves a point $q \in Q_{0}$ in a nilpotent direction determined by the even nilpotent vector $\chi \cdot \eta^{j} \cdot X_{j}$. The last statement is particularly clear in an adapted coordinate system in which the $c^{u}{ }_{j k}$ are absent; in a general chart the $c^{u}{ }_{j k}$ should be seen as an artefact of the coordinate transformation. For every smooth map $\Phi: Q \rightarrow Q$ we have an associated action $\Phi^{*}$ on $\Omega^{1 / 2}{ }_{(c)}(T Q)$ by pull-back: for $\varphi \in \Omega^{1 / 2}{ }_{(c)}(T Q)$ it is defined by

$$
\left(\Phi^{*} \varphi\right)\left((q, \eta) ;\left(e_{j}\right)\right)=\varphi\left(\Phi(q, \eta) ;\left(\Phi_{*} e_{j}\right)\right) \text {. }
$$

Using this we obtain the (super-unitary) action $U$ of $S$ on $\Omega^{1 / 2}{ }_{(c)}(T Q)$ by $U(g, \chi)=\left(\Phi(g, \chi)^{-1}\right)^{*}$; the infinitesimal generators of this action are exactly the operators $\tau(\mathrm{Td})=-i \cdot \mathscr{L}\left(Y_{\mathrm{Td}}\right)$ and $\tau(\Omega)=-i \cdot \mathscr{L}\left(Y_{\Omega}\right)$.

When we have the $S$ action on $\Gamma_{(c)}(\mathrm{QB})^{\mathscr{P}} \cong \Omega^{1 / 2}{ }_{(c)}(T Q)$, we can define $\Gamma_{\text {red }}$ as in (5.33) using the superdeterminant; in view of the relation $\mathrm{gh}=i \cdot \tau(\mathrm{Td})+n / 2$ we obtain the following expression for $\Gamma_{\text {red }}$ (note that the application of the vector field $Y_{\mathrm{Td}}$ does not make sense on $\left.\varphi \in \Omega^{1 / 2}{ }_{(\mathrm{c})}(T Q)\right)$

$$
\begin{aligned}
\Gamma_{\text {red }} & =\left\{\varphi \in \Omega^{1 / 2}(T)(T Q) \mid \forall s \in S: U(s) \varphi=\operatorname{Ber}(s)^{n / 2} \cdot \varphi\right\} \\
& =\left\{\varphi \in \Omega^{1 / 2}{ }_{(c)}(T Q) \mid \mathscr{L}\left(Y_{\mathrm{Td}}\right) \varphi=-(n / 2) \cdot \varphi \& \mathscr{L}\left(Y_{\Omega}\right) \varphi=0\right\} .
\end{aligned}
$$

We are now faced with several questions: (i) does a $\tau(f)$ for $[f] \in H^{0}{ }_{\text {BRST leave }}$ $\Gamma_{\text {red }}$ invariant, (ii) if so, do equivalent functions induce the same operator on $\cdot \Gamma_{\text {red }}$ and (iii) is there any correlation with a direct quantization of the reduced phace space $T^{*} Q_{r}$ ? As we will see, for $\mathscr{P}$-preserving functions the answers are all positive. Given the reduced phase space $T^{*} Q_{r}$ we have the same geometric 
quantization as for any cotangent bundle: the one described above for $T^{*} Q$. Thus the representations space $\Gamma_{(c)}\left(\mathrm{QB}_{r}\right)^{\mathscr{P}_{r}}$ is isomorphic with $\Omega^{1 / 2}{ }_{(c)}\left(T Q_{r}\right)$, a function $f_{r}$ is $\mathscr{P}_{r}$-preserving iff $f_{r}=f_{Q_{r}}+J_{Y_{r}}$ and then $\tau_{r}\left(f_{r}\right)=f_{Q_{r}}-i \cdot \mathscr{L}\left(Y_{r}\right)$. We now claim that $\Gamma_{\text {red }}$ is in a natural way isomorphic to $\Omega^{1 / 2}\left(T Q_{r}\right)$ ! To see this we work with adapted coordinates $(q, \eta)$ on $Q$. If we now define the (local) $1 / 2$-density $v_{t}$ on $Q$ by $v_{t}\left(q, \eta ;\left(\partial / \partial q^{j}, \partial / \partial \eta^{k}\right)\right)=1$, then every $\varphi \in \Omega^{1 / 2}(T Q)$ can be written locally as $\varphi=f \cdot v_{t}$. We now apply $\mathscr{L}\left(Y_{\mathrm{Td}}\right)$ to obtain the condition

$$
\begin{aligned}
\mathscr{L}\left(Y_{\mathrm{Td}}\right) \varphi & =\left(Y_{\mathrm{Td}} f\right) \cdot v_{t}+f \cdot \mathscr{L}\left(Y_{\mathrm{Td}}\right) v_{t} \\
& =\left(\sum_{j} \eta^{j} \cdot \partial f / \partial \eta^{j}\right) \cdot v_{t}+(1 / 2) \cdot \operatorname{Div}_{(\partial / \partial q, \partial / \partial \eta)}\left(Y_{\mathrm{Td}}\right) \cdot f \cdot v_{t} \\
& =-(n / 2) \cdot \varphi \Leftrightarrow \sum_{j} \eta^{j} \cdot \partial f / \partial \eta^{j}=0,
\end{aligned}
$$

where we have used Lemma 2.8 and the fact that $\operatorname{Div}_{(\partial / \partial q, \partial / \partial \eta)}\left(Y_{\mathrm{Td}}\right)=-(n / 2)$. It follows that a $\varphi \in \Gamma_{\text {red }}$ is locally expressed by a function $f$ independent of the coordinates $\eta$. We now apply the same technique to $Y_{\Omega}$, and we note that in adapted coordinates $X_{j}=\partial / \partial q^{j}(j=1, \ldots, n<d)$ and $c^{u}{ }_{j k}=0$. Hence the condition $\mathscr{L}\left(Y_{\Omega}\right) \varphi=0$ translates as

$$
\sum_{j} \eta^{j} \cdot \partial f / \partial q^{j}=0
$$

from which we deduce that $f$ must be the pull-back of a function on $Q_{r}$, which has local coordinates $q^{n+1}, \ldots, q^{d}$. We now define a $1 / 2$-density $\hat{\varphi} \in \Omega^{1 / 2}\left(T Q_{r}\right)$ on the local chart with coordinates $q^{n+1}, \ldots, q^{d}$ by

$$
\hat{\varphi}\left(q^{j>n} ;\left(\partial / \partial q^{j>n}\right)\right)=\varphi\left(q, \eta ;\left(\partial / \partial q^{j}, \partial / \partial \eta^{k}\right)\right) \text {. }
$$

If we introduce the local $1 / 2$-density $v_{t r}$ on $Q_{r}$ defined by $v_{t r}\left(q^{j>n} ;\left(\partial / \partial q^{j>n}\right)\right)=1$, then the map $\Gamma_{\text {red }} \rightarrow \Omega^{1 / 2}\left(T Q_{r}\right), \varphi \rightarrow \hat{\varphi}$ is given by

$$
\varphi=f \cdot v_{t} \rightarrow \hat{\varphi}=f \cdot v_{t r} .
$$

We now have to show that these local expressions glue together to a well-defined global element $\hat{\varphi} \in \Omega^{1 / 2}\left(T Q_{r}\right)$. For this we observe that if $(U, X)$ and $\left(U^{\prime}, X^{\prime}\right)$ are both adapted coordinate charts, then the matrix $\lambda$ connecting the vectors $X$ to $X^{\prime}$ is just part of the Jacobian matrix $\partial q^{\prime} / \partial q$ ! Moreover, denoting by $\mathrm{Jac}_{0}$ the Jacobian of the (full) coordinate transformation $q \rightarrow q^{\prime}$ on $Q_{0}$ and by $\mathrm{Jac}_{r}$ the Jacobian of the (reduced) associated coordinate transformation $q^{n+1}, \ldots, q^{d} \rightarrow q^{\prime n+1}, \ldots, q^{\prime d}$ on $Q_{r}$, we have the equality

$$
\mathrm{Jac}_{0}=\mathrm{Jac}_{r} \cdot \operatorname{Det}(\lambda)
$$

We then compute

$$
\begin{aligned}
\hat{\varphi}\left(q^{j>n} ;\left(\partial / \partial q^{j>n}\right)\right) & =\varphi(q, \eta ;(\partial / \partial q, \partial / \partial \eta)) \\
& =\left|\operatorname{Jac}_{\varphi}\right|^{1 / 2} \cdot \operatorname{Det}(\lambda)^{-1 / 2} \cdot \varphi\left(q^{\prime}, \eta^{\prime} ;\left(\partial / \partial q^{\prime}, \partial / \partial \eta^{\prime}\right)\right) \\
& =\left|\operatorname{Jac}_{r}\right|^{1 / 2} \cdot \hat{\varphi}\left(q^{j>n} ;\left(\partial / \partial q^{j>n}\right)\right),
\end{aligned}
$$

where $\left(q^{\prime}, \eta^{\prime}\right)$ indicates the same point as $(q, \eta)$ and where we have used the fact that our orientability assumption implies that $\operatorname{Det}(\lambda)$ must be positive. It follows from this calculation that $\hat{\varphi}$ is indeed a globally well-defined $1 / 2$-density on $Q_{r}$. The 
reverse procedure shows that to every $\hat{\varphi} \in \Omega^{1 / 2}\left(T Q_{r}\right)$ we can associate a $\varphi \in \Gamma_{\text {red }}$ and thus we have established an isomorphism $\Gamma_{\text {red }} \cong \Omega^{1 / 2}\left(T Q_{r}\right)$.

Remark 6.25. The isomorphism $\Gamma_{\text {red }} \cong \Omega^{1 / 2}\left(T Q_{r}\right)$ obtained above is an identification of $\Omega^{1 / 2}\left(T Q_{r}\right)$ as a subspace of $\Omega^{1 / 2}(T Q)$, where $Q$ is a supermanifold. A similar identification can be obtained without the use of supermanifolds. If we consider $\mathscr{D}_{0}$ as a sub-vector bundle of $T Q_{0}$ over $Q_{0}$, then we obtain $\mathscr{D}_{0}{ }^{*} \rightarrow Q_{0}$ as the dual vector bundle. A computation similar to the (super) calculation described above establishes $\Omega^{1 / 2}\left(T Q_{r}\right)$ as a subspace of $\Omega^{1 / 2}\left(T\left(\mathscr{D}_{0}{ }^{*}\right)\right.$ ). The underlying idea is that, although there is no canonical choice to extend a basis of $T Q_{r}$ to a basis of $T Q_{0}$ by adding vectors of $\mathscr{D}_{0}$, we can extend it in a natural way to a basis of $T\left(\mathscr{D}_{0}{ }^{*}\right)$ by first extending it arbitrarily to a basis of $T Q_{0}$ and then choosing the "dual" basis in $T\left(\mathscr{D}_{0}{ }^{*}\right)$ to extend it to a full basis. The fact that these choices are dual prove that the resulting 1/2-density is well defined. A more detailed account of this idea can be found in [Tu2]; it is the analogue of the result described in [DEGST] for a free group action.

Finally we turn our attention to operators induced on $\Gamma_{\text {red }}$. First we note that two representing functions for $H^{0}{ }_{\text {BRST }}$ are equivalent iff they differ by $\{\Omega, g\}$ with $\{\mathrm{Td}, g\}=-g$. Now if $g$ also preserves $\mathscr{P}$, then we have for $\varphi \in \Gamma_{\text {red }}$ :

$$
\operatorname{gh}(\tau(g) \varphi)=[\operatorname{gh}, \tau(g)] \varphi+\tau(g)(\operatorname{gh}(\varphi))=-\tau(g) \varphi
$$

where we have used Proposition 3.13 and $\mathrm{gh}=i \cdot \tau(\mathrm{Td})+n / 2$. The local analysis of (6.19) shows that the only $\varphi^{\prime} \in \Gamma_{\text {red }}$ satisfying $\operatorname{gh}\left(\varphi^{\prime}\right)=-\varphi^{\prime}$ is the one identically zero, hence $\tau(g) \varphi=0$. From Proposition 3.13 and the definition of $\Gamma_{\text {red }}$ it follows that $\left.\tau(\{\Omega, g\})\right|_{\Gamma_{\text {red }}}=0$ and "thus" equivalent representing functions for $H^{0}{ }_{\text {BRST }}$ induce the same operators on $\Gamma_{\text {red }}$. Now let $f \in C^{\infty}\left(T^{*} Q\right)$ preserve $\mathscr{P}$, i.e., $f=f_{Q}+J_{Y}$, and suppose it represents a class in $H^{0}{ }_{\text {BRST }}$, i.e., $\left\{\mathrm{Td}, f_{Q}+J_{Y}\right\}=0=$ $\left\{\Omega, f_{Q}+J_{Y}\right\}$. Before we translate these conditions to a more concrete form, we need a construction. Let $Y_{0}$ be a vector field on $Q_{0}$ which preserves $\mathscr{D}_{0}$, i.e., $Y_{0}$ projects down to a well-defined vector field $Y_{r}$ on $Q_{r}$, then we define a vector field $Y$ on $Q$ as follows. On a local chart $(U, X)$ we have $\left[X_{j}, Y_{0}\right]=\sum_{k} X_{k} \cdot \mu^{k}{ }_{j}(q)$ and we define $Y$ on this chart by

$$
\left.Y\right|_{(q, \eta)}=\left.Y_{0}\right|_{q}+\sum_{j k} \mu_{j}^{k}(q) \cdot \eta^{j} \cdot \partial / \partial \eta^{k}
$$

With this construction we can describe the $\mathscr{P}$-preserving elements in $H^{0}{ }_{\text {BRST }}$; the proof is an elementary calculation in local charts.

Proposition 6.28. $f=f_{Q}+J_{Y}$ represents a class in $H^{0}{ }_{\text {BRST }}$ if and only if (i) $f_{Q}$ is the pull-back of a function $f_{Q_{r}}$ on $Q_{r}$ by the standard projection $Q \rightarrow Q_{0} \rightarrow Q_{r}$, and (ii) the vector field $Y$ is derived in the above manner from a $\mathscr{D}_{0}$-preserving vector field $Y_{0}$ on $Q_{0}$. If these conditions are satisfied, then the class $\left[f_{Q}+J_{Y}\right] \in H^{0}{ }_{\text {BRST }}$ is mapped to the function $f_{Q_{r}}+J_{Y_{r}} \in C^{\infty}\left(T^{*} Q_{r}\right)$ by the procedure as described following (5.5).

Proposition 6.29. The isomorphism $\Gamma_{\text {red }} \cong \Omega^{1 / 2}\left(T Q_{r}\right)$ intertwines the operator $\tau\left(f_{Q}+J_{Y}\right)$ restricted to $\Gamma_{\text {red }}$ with the operator $\tau_{r}\left(f_{Q_{r}}+J_{Y_{r}}\right)$ on $\Omega^{1 / 2}\left(T Q_{r}\right)$ $\cong \Gamma\left(\mathrm{QB}_{r}\right)^{\mathscr{P}_{r}}$. 
Proof. The part for $f_{Q}$ and $f_{Q_{r}}$ is immediate since it only involves pointwise multiplication of 1/2-densities with functions. For the $J_{Y}$ and $J_{Y_{r}}$ we use adapted coordinates in which the $Y_{0}$ involved is given as $Y_{0}=\sum_{j} y^{j}(q) \cdot \partial / \partial q^{j}$, where the $y^{j}(q)$ for $j>n$ do not depend on $q^{1}, \ldots, q^{n}$ and hence the local expression for $Y_{r}$ is $\sum_{j>n} y^{j}(q) \cdot \partial / \partial q^{j}$. Using the local trivializations $v_{t}$ and $v_{t r}$, the isomorphism $\varphi \rightarrow \hat{\varphi}$ is given by $f \cdot v_{t} \rightarrow f \cdot v_{t r}$ and we calculate:

$$
\begin{aligned}
\mathscr{L}(Y)\left(f \cdot v_{t}\right) & =\left(Y_{r} f\right) \cdot v_{t}+(1 / 2) \cdot \operatorname{Div}_{(\partial / \partial q, \partial / \partial \eta)}(Y) \cdot f \cdot v_{t} \\
& =\left(Y_{r} f\right) \cdot v_{t}+(1 / 2) \cdot \operatorname{Div}_{(\partial / \partial q)}\left(Y_{r}\right) \cdot f \cdot v_{t},
\end{aligned}
$$

where the local expression for $Y$ shows that the divergence of $Y$ with respect to the vectors $\partial / \partial q^{1}, \ldots, \partial / \partial q^{d}, \partial / \partial \eta^{1}, \ldots, \partial / \partial \eta^{n}$ equals the divergence of $Y_{r}$ with respect to the vectors $\partial / \partial q^{n+1}, \ldots, \partial / \partial q^{d}$ (the contributions of the remaining $\partial / \partial q$ 's cancel those of the $\partial / \partial \eta$ 's). Hence with $\mathscr{L}\left(Y_{r}\right) v_{t r}=(1 / 2) \cdot \operatorname{Div}_{(\partial / \partial q)}\left(Y_{r}\right) \cdot v_{t r}$ we conclude that $\widehat{\left(\mathscr{L}(Y)\left(f \cdot v_{t}\right)\right)}=\mathscr{L}\left(Y_{r}\right)\left(\left(\widehat{\left.f \cdot v_{t}\right)}\right)\right.$, which finishes the proof.

Remark 6.31. Our approach is different from the approach by McMullan and Paterson [MP] in that we do not use so-called self-dual elements. This has a definite advantage since one needs in the general case additional structure (i.e. a metric on $\mathscr{D}_{0}$ ) in order to define self-duality.

Acknowledgement. I would like to thank M. Gotay and R. Schmid for many stimulating and clarifying discussions. I also would like to thank the MSRI (Berkeley) for the hospitality they offered me while this research was done. Finally I would like to thank the referee whose comments have improved the exposition of this paper.

\section{References}

[AGJ] Arms, J.M., Gotay, M.J., Jennings, G.: Geometric and algebraic reduction for singular momentum maps. Adv. in Math. 79, 43-103 (1990)

[Ba1] Batchelor, M.: The structure of supermanifolds. Trans. AMS 253, 329-338 (1979)

[Ba2] Batchelor, M.: Graded manifolds and supermanifolds. In: Mathematical aspects of super space, pp. 91-133, Seiffert, H., et al. (eds.), Dordrecht: Reidel 1984

[BM] Browning, A.D., McMullan, D.: The Batalin, Fradkin, and Vilkovisky formalism for higher-order theories. J. Math. Phys. 28, 438-444 (1987)

[DET] Duval, C., Elhadad, J., Tuynman, G.M.: The BRS method and geometric quantization: Some examples. Commun. Math. Phys. 126, 535-557 (1990)

[DEGST] Duval, C., Elhadad, J., Gotay, M.J., Śniatycki, J., Tuynman, G.M.: Quantization and Bosonic BRS theory. Ann. Phys. 206, 1-26 (1991)

[DEGT] Duval, C., Elhadad, J., Gotay, M.J., Tuynman, G.M.: Nonunimodularity and the quantization of the pseudo-rigid-body. In: Hamiltonian systems, transformation groups and spectral transform methods, Harnad, J., Marsden, J.E. (eds.), pp. 149-160. Montreal: Publ. C.R.M. 1990

[dW] DeWit, B.S.: Supermanifolds. Cambridge: Cambridge Univ. Press 1984

[FHST] Fisch, J., Henneaux, M., Stasheff, J., Teitelboim, C.: Existence, uniqueness and cohomology of the classical BRST charge with ghosts of ghosts. Commun. Math. Phys. 120, 379-407 (1988)

[Go] Gotay, M.J.: Constraints, reduction and quantization. J. Math. Phys. 27, 2051-2066 (1986)

[GS] Guillemin, V., Sternberg, S.: Geometric quantization and multiplicities of group representations. Invent. Math. 67, 515-538 (1982)

[He] Henneaux, M.: Classical foundations of BRST symmetry. Napoli: Bibliopolis, Napoli, 1988. (Monographs and textbooks in physical sciences lecture notes no 7) 
[HT] Henneaux, M., Teitelboim, C.: BRST cohomology in classical mechanics. Commun. Math. Phys. 115, 213-230 (1988)

[Ko1] Kostant, B.: Quantization and unitary representations. In: Lectures in modern analysis and applications III, pp. 87-208 (Taam, C.T. (ed.) Lecture Notes in Mathematics, vol. 170. Berlin, Heidelberg, New York: Springer 1970

[Ko2] Kostant, B.: Graded manifolds, graded Lie theory and prequantization. In: Differential geometric methods in mathematical physics, pp. 177-306. Lecture Notes in Mathematics, vol. 570. Berlin, Heidelberg, New York: Springer 1977

[KS] Kostant, B., Sternberg, S.: Symplectic reduction, BRS cohomology, and infinitedimensional Clifford algebras. Ann. Phys. 176, 49-113 (1987)

$[\mathrm{Ku}]$ Kuchař, K.: Hamiltonian dynamics of gauge systems/Covariant factor ordering of gauge systems. Phys. Rev. D 34, 3031-3043/3044-3057 (1986)

[Le] Leites, D.A.: Introduction to the theory of supermanifolds. Russ. Math. Surv. 35, 1-64 (1980)

[Lo] Loll, R.: The extended phase space of the BRS approach. Commun. Math. Phys. 119, 509-527 (1988)

[MP] McMullan, D., Paterson, J.: Covariant factor ordering of gauge systems using ghost variables I \& II. J. Math. Phys. 30, 477-497 (1989)

[MW] Marsden, J., Weinstein, A.: Reduction of symplectic manifolds with symmetry. Rep. Math. Phys. 5, 121-130 (1974)

[Ro] Rothstein, M.J.: Integration on noncompact supermanifolds. Trans. AMS 299, 387396 (1987)

[Sh] Shander, V.N.: Orientations of supermanifolds. Funct. Anal. Appl. 22, 80-82 (1988)

[Sn] Śniatycki, J.: Geometric quantization and quantum mechanics. Berlin, Heidelberg, New York: Springer 1980. (Appl. Math. Sci. 30)

[So] Souriau, J.M.: Structure des systèmes dynamiques. Paris: Dunod 1969

[Sta1] Stasheff, J.: Constrained Poisson algebras and strong homotopy representations. Bull. AMS 19, 287-290 (1988)

[Sta2] Stasheff, J.: Homological reduction of constrained Poisson algebras. Preprint UNCMath, Chapel Hill, NC 27514

[SW] Simms, D.J., Woodhouse, N.: Lectures on geometric quantization. Lecture Notes in Physics, vol. 53. Berlin, Heidelberg, New York: Springer 1977

[Tu1] Tuynman, G.M.: Proceedings Seminar 1983-1985 mathematical structures in field theories, Vol. I: Geometric quantization. Amsterdam: CWI 1985 (CWI syllabus 8)

[Tu2] Tuynman, G.M.: Quantization of first class constraints with structure functions. Lett. Math. Phys. 21, 205-213 (1991)

[Wo] Woodhouse, N.: Geometric quantization. Oxford: Oxford University Press 1980

Communicated by A. Jaffe 
